\title{
Achievable rate regions and performance comparison of half duplex bi-directional relaying protocols
}

\author{
Sang Joon Kim, Natasha Devroye, Patrick Mitran, and Vahid Tarokh
}

\begin{abstract}
In a bi-directional relay channel, two nodes wish to exchange independent messages over a shared wireless half-duplex channel with the help of a relay. In this paper, we derive achievable rate regions for four new half-duplex protocols and compare these to four existing half-duplex protocols and outer bounds. In time, our protocols consist of either two or three phases. In the two phase protocols, both users simultaneously transmit during the first phase and the relay alone transmits during the second phase, while in the three phase protocol the two users sequentially transmit followed by a transmission from the relay. The relay may forward information in one of four manners; we outline existing Amplify and Forward (AF), Decode and Forward (DF), Lattice based, and Compress and Forward (CF) relaying schemes and introduce the novel Mixed Forward scheme. The latter is a combination of CF in one direction and DF in the other. We derive achievable rate regions for the CF and Mixed relaying schemes for the two and three phase protocols. We provide a comprehensive treatment of eight possible half-duplex bi-directional relaying protocols in Gaussian noise, obtaining their relative performance under different SNR and relay geometries.
\end{abstract}

\section{Index Terms}

Sang Joon Kim and Vahid Tarokh are with the School of Engineering and Applied Sciences, Harvard University, Cambridge, MA 02138. Emails: adella0919@gmail.com, vahid@seas.harvard.edu. Natasha Devroye is with the Department of Electrical and Computer Engineering, University of Illinois at Chicago, Chicage, IL 60607. Email: devroye@ece.uic.edu. Patrick Mitran is with the Department of Electrical and Computer Engineering, University of Waterloo, Waterloo, Canada. Email: pmitran@ecemail.uwaterloo.ca. This research is supported in part by NSF grant number ACI-0330244 and ARO MURI grant number W911NF-07-1-0376. This work was supported in part by the Army Research Office, under the MURI award N0. N00014-01-1-0859. The views expressed in this paper are those of the author alone and not of the sponsor. 
bi-directional communication, achievable rate regions, compress and forward, relaying

\section{INTRODUCTION}

Bi-directional relay channels, or wireless channels in which two nodes ( $a$ and $b)^{1}$ wish to exchange independent messages with the help of a third relay node $r$, are both of theoretical and practical interest. Such channels may be relevant to ad hoc networks as well as to networks with a centralized controller through which all messages must pass. From an information theoretic perspective, an understanding of these fundamental bi-directional channels would bring us closer to a coherent picture of multi-user information theory. To this end, we study bi-directional relay channels with the goal of determining spectrally efficient achievable rate regions and tight outer bounds to the capacity region. In this work, we consider half-duplex communication in which a node may either transmit or receive at a given moment, but not both. This is in contrast to full-duplex operation where nodes transmit and receive on the same antenna and frequency simultaneously. Unfortunately, full-duplex operation may not be practically feasible as the intensity of the near field of the transmitted signal is much higher than that of the far field of the received signal, motivating the consideration of half-duplex operation. Our goal is to determine spectrally efficient (measured in bits per channel use) transmission schemes and outer bounds for the half-duplex bi-directional relay channel and compare their performance in a number of scenarios. These scenarios highlight the fact that different protocols may be optimal under different channel conditions. An obvious half-duplex bi-directional relay protocol is the four phase protocol, $a \rightarrow r, r \rightarrow b, b \rightarrow r$ and $r \rightarrow a$, where the phases are listed chronologically. However, this protocol is spectrally inefficient and does not take full advantage of the broadcast nature of the wireless channel. One way to take advantage of the shared wireless medium is to combine the second and the fourth phases into a single broadcast transmission by using, for example, network coding [1]. That is, if the relay $r$ can decode the messages $w_{\mathrm{a}}$ and $w_{\mathrm{b}}$ from nodes $\mathrm{a}$ and $\mathrm{b}$ respectively, it is sufficient for the relay $\mathrm{r}$ to broadcast $w_{\mathrm{a}} \oplus w_{\mathrm{b}}$ to both $\mathrm{a}$ and $\mathrm{b}$. Alternative capacity achieving strategies for this second downlink phase alone are proposed using tailored binning strategies in [12], [22], [32]. In this paper we consider two possible bi-directional relay protocols which differ in their number of phases. Throughout this work, phases will denote temporal phases, or durations. The three phase protocol is called the Time Division Broadcast (TDBC) protocol, while the two phase protocol is called the Multiple Access Broadcast (MABC) protocol. One of the main conceptual differences between these two protocols is the possibility of side-information in the TDBC protocol but

\footnotetext{
${ }^{1}$ We call the nodes a and b terminal and source nodes interchangeably.
} 
not in the MABC protocol. By side-information we mean information obtained from the wireless channel in a particular phase which may be combined with information obtained in different stages to potentially improve decoding or increase transmission rates. The two considered protocols may be described as:

1) TDBC protocol: this consists of the three phases $a \rightarrow r, b \rightarrow r$ and $a \leftarrow r \rightarrow b$; only a single node is transmitting during a given phase. By the broadcast nature of the wireless channel, the non-transmitting nodes may listen in and obtain "side information" about the transmissions of the other nodes, which in turn may improve rates.

2) MABC protocol: this protocol combines the first two phases of the TDBC protocol and consists of the two phases $\mathrm{a} \rightarrow \mathrm{r} \leftarrow \mathrm{b}$ and $\mathrm{a} \leftarrow \mathrm{r} \rightarrow \mathrm{b}$. Due to the half-duplex assumption, during phase 1 both source nodes are transmitting and thus cannot obtain any "side information" regarding the other nodes' transmission. It may nonetheless be spectrally efficient since it has less phases than the TDBC protocol and may take advantage of the multiple-access channel in phase 1.

We consider restricted protocols in the sense that the receivers must decode their messages at the end of the third phase (TDBC) or second phase (MABC) and collaboration across multiple successive runs of the protocols are not possible. For each of the MABC and TDBC protocols, the relay may process and forward the received signals differently. These different forwarding schemes are motivated by different relaying capabilities or assumptions (about the required complexity or knowledge). Combining the relaying schemes with the temporal protocols, we can obtain various protocols whose rate regions are not in general subsets of one another. The relative benefits and merits of the four relaying schemes are summarized in Table I. The five relaying schemes we consider are:

1) Amplify and Forward (AF): the relay $r$ constructs its symbol by symbol replication of the received symbol. The AF scheme does not require any computation for relaying except for simple symbol based addition, and carries noise incurred in the first stage(s) forward during the relaying stage.

2) Decode and Forward (DF): the relay decodes both messages from nodes a and b before re-encoding them for transmission. The DF scheme requires the full codebooks of both $a$ and $b$ and a large amount of computation at the relay $r$.

3) Compress and Forward (CF): the relay does not decode the messages of a and b, nor does it simply amplify the received signal, but it performs something in between these two extremes. It compresses the received signal, which it then transmits. To do so, the relay does not require the codebooks of the source nodes, but it does require the channel output distribution $p\left(y_{r}\right)$ at the relay. 
4) Mixed Forward: the relay decodes and forwards (DF) the data traveling in one direction $(a \rightarrow b)$, while it compresses and forwards $(\mathrm{CF})$ the data traveling in the other direction $(\mathrm{a} \leftarrow \mathrm{b})$. For the mixed scheme, one of the codebooks and the channel output distribution are needed at the relay.

5) Lattice Forward (for Gaussian noise channels only): for MABC protocols where a multiple access channel exists in the first phase, it may be more spectrally efficient to directly decode and forward a linear combination of the transmitted codewords. By employing structured lattice codes [20], [33], one may exploit the linear relationship between channel inputs and outputs to decode a sum of the codewords rather than individual codewords as is the case when using random codebooks.

A large portion of the results presented will focus on the CF-forwarding scheme, which is seen as an alternative to DF forwarding that is slightly less computationally expensive and may lead to increased rates due to the lack of decoding required at relay nodes. In the $\mathrm{CF}$ scheme the relay searches the compression codebook to find an appropriate codeword: this is similar to the decoding operation in the DF scheme, but the CF scheme's complexity is controllable by the choice of the CF codebook. Some of these protocols and relaying schemes have been considered in the past. Very little work has considered the three phase TDBC protocol; only [13] and [15] have considered the DF TDBC protocol. In the latter, network coding in $\mathbb{Z}_{2}^{k}$ is used to encode the message of relay $r$ from the estimated messages $\tilde{w}_{\mathrm{a}}$ and $\tilde{w}_{\mathrm{b}}$. In contrast, the 2-phase MABC protocols have been much more thoroughly considered. The works of [24] and [25] consider the MABC protocol with an "amplify and denoise" relaying scheme. In [17], [18] lattice codes are used at the terminal nodes for the Gaussian channel in the MABC protocol, allowing the relay to decode a combination of the transmitted messages. The capacity region of the broadcast phase in the $\mathrm{MABC}$ protocol, assuming the relay has both messages $w_{\mathrm{a}}$ and $w_{\mathrm{b}}$ (and each terminal node has its own message side information) is found in [12], [21], [32]. In [31] and [19] Slepian-Wolf coding is extended to lossy broadcast channels with side information at the receivers. In [13], achievable rate regions and outer bounds of the MABC protocol and the TDBC protocol with the DF relaying scheme are derived. In this work we place a particular focus on CF relaying schemes as well as on the numerical comparison of different schemes in AWGN. Uni-directional CF relaying in the full-duplex channel is first introduced in [5]. An achievable region in the CF MABC protocol is derived in [27] and enhanced in [29], while an achievable region of the partial DF MABC protocol is derived in [28]. In [9] a comparison between DF and CF schemes in full-duplex channels is performed, while in [26] a comparison of AF and DF schemes with two relays in the MABC protocol is performed. In this paper, we derive achievable regions for new $\mathrm{CF}$ and mixed relaying schemes in both the TDBC and MABC half-duplex protocols. 
TABLE I

COMPARISON BETWEEN FOUR RELAYING SCHEMES

\begin{tabular}{c||c|c|c}
\hline Relaying & Complexity & Noise at relay & Relay needs \\
\hline AF & very low & carried plus noise at rx & nothing \\
DF & high & perfectly eliminated & full codebooks \\
CF & low & carried plus distortion & $p\left(y_{\mathrm{r}}\right)$ \\
Mixed & moderate & partially carried & one codebook, $p\left(y_{\mathrm{r}}\right)$ \\
Lattice (Gaussian only) & high & eliminated, decode the sum of codewords & full codebooks \\
\hline
\end{tabular}

We also obtain outer bounds for the TDBC and MABC protocols based on cut-set bounds. We compare the achievable rate regions of the novel schemes with the regions and outer bounds derived in [13] as well as a simple AF scheme, and an extension of the lattice-based schemes (previously presented for fullduplex channels) to half-duplex channels in Gaussian noise. We thus present a comprehensive overview of the bi-directional relay channel which highlights the relative performance and tradeoffs of the different schemes under different channel conditions and relay processing capabilities. Notably, we find that under some channel conditions the mixed TDBC protocol outperforms the other protocols and similarly, there are channel conditions for which the CF TDBC protocol has the best performance. We also see that in the high SNR region the sum rate of the Lattice DF MABC protocol is very close to the outer bound; we show that this gap may be bounded by 1 bit.

This paper is structured as follows: in Section II, we introduce our notation. In Section III we derive achievable rate regions for the $\mathrm{CF}$ and mixed relaying schemes. In Section IV we obtain explicit expressions for these, new lattice based, and previous rate regions and outer bounds in Gaussian noise. In Section V, we numerically compute these bounds in the Gaussian noise channel and compare the results for different powers and channel conditions.

\section{PRELIMINARIES}

In this work, we will determine and compare the rate regions of eight bi-directional half-duplex relay protocols. We will mainly consider the less-studied 3 phase Time Division Broadcast (TDBC) protocol as well as provide a slight generalization of the 2 phase Multiple Access Broadcast (MABC) versions of Amplify and Forward (AF), Decode and Forward (DF), Compress and Forward (CF), Lattice forwarding (for the Gaussian channel under MABC protocol only), as well as a Mixed scheme which combines Decode and Forward in one direction with Compress and Forward in the other. The AF, DF protocol 
regions and the $\mathrm{CF}$ MABC protocol region have been derived in prior work [13], [25], [27] while an improvement of the [27] CF MABC, the CF TDBC, Lattice MABC and Mixed MABC and TDBC protocol regions described in Section III are determined here.

\section{A. Notation and Definitions}

We consider two terminal nodes a and $\mathrm{b}$, and one relay node $\mathrm{r}$. Terminal node a has a message $W_{\mathrm{a}}$ uniformly distributed in $\left\{0, \ldots,\left\lfloor 2^{n R_{\mathrm{a}}}\right\rfloor-1\right\}=: \mathcal{S}_{\mathrm{a}}$ to be decoded at node $\mathrm{b}$ at rate $R_{\mathrm{a}}$. Node $\mathrm{b}$ has an independent message $W_{\mathrm{b}}$ uniformly distributed in $\left\{0, \ldots,\left\lfloor 2^{n R_{\mathrm{b}}}\right\rfloor-1\right\}=: \mathcal{S}_{\mathrm{b}}$ to be decoded at node a at rate $R_{\mathrm{b}}$. The relay node $\mathrm{r}$ may assist in the bi-directional endeavor. The nodes are assumed to be halfduplex, which implies that they cannot simultaneously transmit and receive data. As a result, achievability schemes are protocol dependent; a protocol defines which nodes transmit during each temporal phase. The protocols considered have either 2 (MABC) or 3 (TDBC) phases. The relative time duration of the $\ell^{\text {th }}$ phase is denoted by $\Delta_{\ell} \geq 0$, where $\sum_{\ell} \Delta_{\ell}=1$. For a given block size $n, \Delta_{\ell, n}$ denotes the normalized (by $n$ ) duration of the $\ell^{t h}$ phase, and in achievability schemes we will require $\lim _{n \rightarrow \infty} \Delta_{\ell, n}=\Delta_{\ell}$. The channel input and output at channel use $k$ at node $i$ are denoted by the random variables $X_{i}^{k} \in \mathcal{X}_{i}$ and $Y_{i}^{k} \in \mathcal{Y}_{i}$ respectively, for $i \in\{\mathrm{a}, \mathrm{b}, \mathrm{r}\}$. Channel inputs are related to channel outputs according to a discrete memoryless channel. We note that the distributions of $X_{i}^{k}$ and $Y_{i}^{k}$ depend on the value of $k$, e.g. for $k \leq \Delta_{1, n} \cdot n$ we are in phase 1 , for $\Delta_{1, n} n<k \leq\left(\Delta_{1, n}+\Delta_{2, n}\right) n$ we are in phase 2 and for $\left(\Delta_{1, n}+\Delta_{2, n}\right) n<k \leq n$ we are in phase 3 (in TDBC protocols only). With a slight abuse of notation, we use $X_{i}^{(\ell)}$ to denote the random variable with alphabet $\mathcal{X}_{i}$ and input distribution $p^{(\ell)}\left(x_{i}\right)$ during phase $\ell$. As multiple nodes may transmit during a particular phase, we let $X_{S}^{k}:=\left\{X_{i}^{k} \mid i \in S\right\}$ denote the set of transmissions by all nodes in the set $S$ at time $k$, and let $X_{S}^{(\ell)}:=\left\{X_{i}^{(\ell)} \mid i \in S\right\}$ denote a set of random variables with channel input distribution $p^{(\ell)}\left(x_{S}\right)$ for phase $\ell$, where $x_{S}:=\left\{x_{i} \mid i \in S\right\}$. Lower case letters $x_{i}$ will denote instances of the upper case $X_{i}$ which lie in the calligraphic alphabets $\mathcal{X}_{i}$. Boldface $\mathbf{x}_{i}$ represents a vector indexed by time at node $i$. Finally, let $\mathbf{x}_{S}:=\left\{\mathbf{x}_{i} \mid i \in S\right\}$ denote a set of vectors corresponding to nodes in the set $\mathcal{S}$ indexed by time. We will be constructing Compress and Forward schemes in which received signals are compressed or quantized before being re-transmitted. $\hat{Y}_{i}$ denotes the compressed representation of the received signal at node $i$, which lies in the corresponding compression alphabet $\hat{\mathcal{Y}}_{i}$ for node $i . \hat{\mathcal{Y}}_{i}$ is not necessarily equal to $\mathcal{Y}_{i}$. However, in our numerical Gaussian results in Sections IV and V, $\mathcal{X}_{i}=\mathcal{Y}_{i}=\hat{\mathcal{Y}}_{i}=\mathbb{C}, \forall i$. Encoders, decoders and associated probability of errors are defined as follows: let $W_{S, T}:=\left\{W_{i, j} \mid i \in S, j \in T, S, T \subset \mathcal{M}\right\}$ denote the set of messages from nodes in set $S$ to nodes in set $T$. We note that if node $i$ does not have a message for node $j$, 
then $W_{i, j}=\emptyset$. The encoder at node $i$ at channel use $k$ is a function $X_{i}^{k}\left(W_{\{i\}, \mathcal{M}}, Y_{i}^{1}, \cdots, Y_{i}^{k-1}\right) \in \mathcal{X}_{i}$; the decoder at node $i$ after all $n$ channel uses is a function $\tilde{W}_{j, i}\left(Y_{i}^{1}, \cdots, Y_{i}^{n}, W_{\{i\}, \mathcal{M}}\right)$ which produces an estimate of the message $W_{j, i}$. We define error events $E_{i, j}:=\left\{W_{i, j} \neq \tilde{W}_{i, j}().\right\}$ for decoding the message $W_{i, j}$ at node $j$ at the end of the block of length $n$, and $E_{i, j}^{(\ell)}$ as the error event at node $j$ in which node $j$ attempts to decode $w_{i}$ at the end of phase $\ell$. Let $A^{(\ell)}(U V)$ represent the set of $\epsilon$-typical $\left(\mathbf{u}^{(\ell)}, \mathbf{v}^{(\ell)}\right)$ sequences of length $n \cdot \Delta_{\ell, n}$ according to the distributions $U$ and $V$ in phase $\ell$ and let $D^{(\ell)}(\mathbf{u}, \mathbf{v}):=\left\{\left(\mathbf{u}^{(\ell)}, \mathbf{v}^{(\ell)}\right) \in A^{(\ell)}(U V)\right\}$ denote the event that $\mathbf{u}$ and $\mathbf{v}$ are jointly typical. In general, joint typicality is non-transitive. However, by using strong joint-typicality, and the fact the distributions of interest will generally form Markov chains $X \rightarrow Y \rightarrow \hat{Y}$ we will be able to argue joint typicality between $\mathbf{x}$ and $\hat{\mathbf{y}}$ by the Markov lemma of Lemma 4.1 in [2] and the extended Markov lemma (Lemma 3 of [23], Remark 30 of [14]).

A set of rates $R_{i, j}$ is said to be achievable for a protocol with phase durations $\left\{\Delta_{\ell}\right\}$ if there exist encoders/decoders of block length $n=1,2, \ldots$ with both $P\left[E_{i, j}\right] \rightarrow 0$ and $\Delta_{\ell, n} \rightarrow \Delta_{\ell}$ as $n \rightarrow \infty$ for all $i, j, \ell$. An achievable rate region (resp. capacity region) is the closure of a set of (resp. all) achievable rate tuples for fixed $\left\{\Delta_{\ell}\right\}$. Finally, we let $Q$ denote a discrete time-sharing random variable with distribution $p(q)$ and let $\bar{A}$ denote the complement of the set $A .[x]^{+}:=\max \{x, 0\}$ for $x \in \mathbb{R}$.

\section{B. Compress and Forward using two joint typicality decoders}

In Compress and Forward protocols, unlike in Decode and Forward protocols, the relay node $r$ does not decode the message $w_{\mathrm{a}}$ or $w_{\mathrm{b}}$. Thus, network coding techniques such as the algebraic group operation $w_{\mathrm{a}} \oplus w_{\mathrm{b}}$ used in [13] cannot be used to generate $w_{\mathrm{r}}$ for the current $\mathrm{CF}$ schemes. Instead, two jointly typical decoders at each node are used to decode $w_{\mathrm{r}}$.

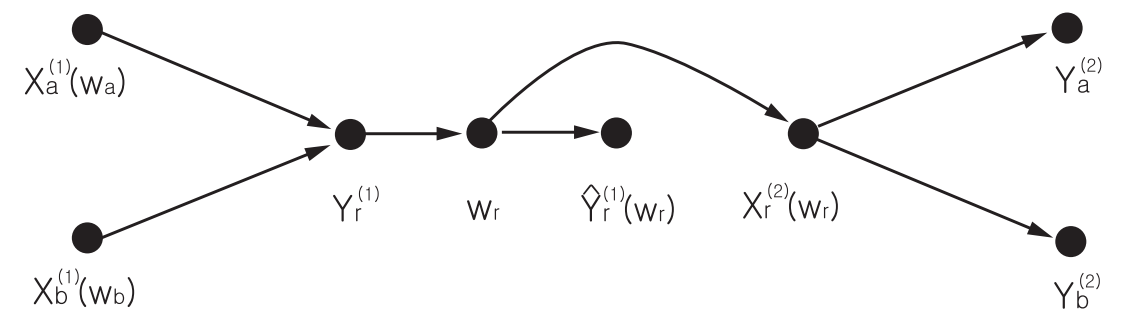

Fig. 1. The data flow in the compress and forward MABC protocol

To illustrate this in the MABC protocol, consider the decoder at node a which wishes to decode the relay message $w_{\mathrm{r}}$ in order to ultimately decode the desired message from node $\mathrm{b}, w_{\mathrm{b}}$. After phase 2 , node a has 
the known sequences $\mathbf{x}_{\mathrm{a}}^{(1)}\left(w_{\mathrm{a}}\right)$ and $\mathbf{y}_{\mathrm{a}}^{(2)}$. Node a then finds the set of all $\hat{\mathbf{y}}_{\mathrm{r}}^{(1)}\left(w_{\mathrm{r}}\right)$ and $\mathbf{x}_{\mathrm{r}}^{(2)}\left(w_{\mathrm{r}}\right)$ such that $\left(\mathbf{x}_{\mathrm{a}}^{(1)}\left(w_{\mathrm{a}}\right), \hat{\mathbf{y}}_{\mathrm{r}}^{(1)}\left(w_{\mathrm{r}}\right)\right)$ and $\left(\mathbf{x}_{\mathrm{r}}^{(2)}\left(w_{\mathrm{r}}\right), \mathbf{y}_{\mathrm{a}}^{(2)}\right)$ are two pairs of jointly typical sequences, as shown in Fig. 1. Node a then decodes $w_{\mathrm{r}}$ correctly if there exists a unique $w_{\mathrm{r}}$ such that $\left(\mathbf{x}_{\mathrm{a}}^{(1)}\left(w_{\mathrm{a}}\right), \hat{\mathbf{y}}_{\mathrm{r}}^{(1)}\left(w_{\mathrm{r}}\right)\right) \in A^{(1)}\left(X_{\mathrm{a}} \hat{Y}_{\mathrm{r}}\right)$ and $\left(\mathbf{x}_{\mathrm{r}}^{(2)}\left(w_{\mathrm{r}}\right), \mathbf{y}_{\mathrm{a}}^{(2)}\right) \in A^{(2)}\left(X_{\mathrm{r}} Y_{\mathrm{a}}\right)$ and declares a decoding error otherwise.

\section{ACHIEVAble RATE REgions For COMPRESS AND Forward AND MiXed Protocols}

In this Section we present three new achievable rate regions in Theorems 1 (3-phase CF TDBC) and 2 (3-phase Mixed TDBC), and a slight improvement of [27] in Theorem 3 (2-phase CF MABC). As an aside, we provide the negative result that the logical extension of the 2-phase MABC protocol to a Mixed forwarding scheme (DF in one direction and CF in the other) always lies within the DF MABC region of [13]. These regions, derived here for the discrete memoryless channel, will be extended to the Gaussian noise channel in sections IV and V, where we present an additional 2-phase achievable MABC region which exploits structured lattice codes to decode sums of messages.

\section{A. TDBC Protocol}

Our main results in this section are the derivation of two new achievable rate regions for the 3-phase TDBC protocol: one using CF in both directions, and one using CF in one direction and DF in the other, which we term "Mixed" forwarding. In phase 1 and 2, each of the terminal nodes transmits. During phase 3, the "relay broadcast" phase, the relay transmits in two sub-phases - separated in time due to simplicity. In the first relay-broadcast sub-phase, we use the Marton-broadcast-like scheme [16], in which two different messages are transmitted to the two receivers. In this scheme, neither receiver uses side information ( $w_{\mathrm{a}}$ at node $\mathrm{a}$ and $w_{\mathrm{b}}$ at node $\mathrm{b}$ ) to decode the messages. In the second relay-broadcasting phase, we assume a compound channel, i.e., a common message is transmitted to the two receivers which have different side information. Because we use two sub-phases, different proportions of the messages $W_{\mathrm{a}}$ and $W_{\mathrm{b}}$ are transmitted during the two sub-phases. We let $\alpha_{\mathrm{a}}$ (resp. $\alpha_{\mathrm{b}}$ ) denote the fraction of the information content of $W_{\mathrm{a}}$ (resp. $W_{\mathrm{b}}$ ) transmitted by Marton-broadcasting-like scheme; the remainder is broadcast in the compound-channel-like scheme. For convenience of analysis, we denote the first part of the relay-broadcast phase as phase 3 and the second as phase 4. During phase 1 , node a sends $w_{\mathrm{a}}$ using codeword $\mathbf{x}_{\mathrm{a}}^{(1)}\left(w_{\mathrm{a}}\right)$. Since node $\mathbf{b}$ is silent, direct-link side information is available at node $\mathbf{b}$. The relay receives the signal $\mathbf{y}_{\mathrm{r}}^{(1)}$ according to $p^{(1)}\left(y_{\mathrm{r}} \mid x_{\mathrm{a}}\right)$ and compresses it to a signal $\hat{\mathbf{y}}_{\mathrm{r}}^{(1)}\left(w_{\mathrm{a} 0}\right)$ with the index $w_{\mathrm{a} 0}$. A similar process is performed during phase 2 in sending message $w_{\mathrm{b}}$ from node $\mathrm{b}$ to node a with the help of the relay which compresses the received signal to the index $w_{\mathrm{b}, 0}$. Then the relay 
broadcasts a portion $\left(\alpha_{\mathrm{a}}\right.$ and $\left.\alpha_{\mathrm{b}}\right)$ of the compression messages $w_{\mathrm{a} 0}$ and $w_{\mathrm{b} 0}$ using a scheme similar to that in Marton's broadcast channel region during phase 3 and sends a common message as in a compound channel during phase 4 . The main challenge lies in finding the optimum compression strategies and ratios between two relay broadcasting schemes by exploiting the terminal nodes' own messages and direct-link side information.

Theorem 1: An achievable rate region of the half-duplex bi-directional relay channel with the compress and forward TDBC protocol is the closure of the set of all points $\left(R_{\mathrm{a}}, R_{\mathrm{b}}\right)$ satisfying

$$
\begin{aligned}
& R_{\mathrm{a}}<\Delta_{1} I\left(X_{\mathrm{a}}^{(1)} ; \hat{Y}_{\mathrm{r}}^{(1)}, Y_{\mathrm{b}}^{(1)} \mid Q\right) \\
& R_{\mathrm{b}}<\Delta_{2} I\left(X_{\mathrm{b}}^{(2)} ; \hat{Y}_{\mathrm{r}}^{(2)}, Y_{\mathrm{a}}^{(2)} \mid Q\right)
\end{aligned}
$$

subject to

$$
\begin{aligned}
& \alpha_{\mathrm{a}} \Delta_{1} I\left(Y_{\mathrm{r}}^{(1)} ; \hat{Y}_{\mathrm{r}}^{(1)} \mid Q\right)<\Delta_{3} I\left(U_{\mathrm{a}}^{(3)} ; Y_{\mathrm{b}}^{(3)} \mid Q\right) \\
& \alpha_{\mathrm{b}} \Delta_{2} I\left(Y_{\mathrm{r}}^{(2)} ; \hat{Y}_{\mathrm{r}}^{(2)} \mid Q\right)<\Delta_{3} I\left(U_{\mathrm{b}}^{(3)} ; Y_{\mathrm{a}}^{(3)} \mid Q\right) \\
& \alpha_{\mathrm{a}} \Delta_{1} I\left(Y_{\mathrm{r}}^{(1)} ; \hat{Y}_{\mathrm{r}}^{(1)} \mid Q\right)+\alpha_{\mathrm{b}} \Delta_{2} I\left(Y_{\mathrm{r}}^{(2)} ; \hat{Y}_{\mathrm{r}}^{(2)} \mid Q\right)<\Delta_{3} I\left(U_{\mathrm{a}}^{(3)} ; Y_{\mathrm{b}}^{(3)} \mid Q\right)+\Delta_{3} I\left(U_{\mathrm{b}}^{(3)} ; Y_{\mathrm{a}}^{(3)} \mid Q\right)-\Delta_{3} I\left(U_{\mathrm{a}}^{(3)} ; U_{\mathrm{b}}^{(3)} \mid Q\right)
\end{aligned}
$$$$
\left(1-\alpha_{\mathrm{a}}\right) \Delta_{1} I\left(Y_{\mathrm{r}}^{(1)} ; \hat{Y}_{\mathrm{r}}^{(1)} \mid Q\right)+\Delta_{2} I\left(Y_{\mathrm{r}}^{(2)} ; \hat{Y}_{\mathrm{r}}^{(2)} \mid X_{\mathrm{b}}^{(2)}, Q\right)<\Delta_{4} I\left(X_{\mathrm{r}}^{(4)} ; Y_{\mathrm{b}}^{(4)}\right)+\Delta_{1} I\left(\hat{Y}_{\mathrm{r}}^{(1)} ; Y_{\mathrm{b}}^{(1)} \mid Q\right)
$$$$
\left(1-\alpha_{\mathrm{b}}\right) \Delta_{2} I\left(Y_{\mathrm{r}}^{(2)} ; \hat{Y}_{\mathrm{r}}^{(2)} \mid Q\right)+\Delta_{1} I\left(Y_{\mathrm{r}}^{(1)} ; \hat{Y}_{\mathrm{r}}^{(1)} \mid X_{\mathrm{a}}^{(1)}, Q\right)<\Delta_{4} I\left(X_{\mathrm{r}}^{(4)} ; Y_{\mathrm{a}}^{(4)}\right)+\Delta_{2} I\left(\hat{Y}_{\mathrm{r}}^{(2)} ; Y_{\mathrm{a}}^{(2)} \mid Q\right)
$$

where $0<\alpha_{\mathrm{a}}, \alpha_{\mathrm{b}}<1$ over all joint distributions,

$$
\begin{aligned}
& p\left(q, x_{\mathrm{a}}, x_{\mathrm{b}}, x_{\mathrm{r}}, y_{\mathrm{a}}, y_{\mathrm{b}}, y_{\mathrm{r}}, \hat{y}_{\mathrm{r}}\right) \\
& \quad=p(q) p^{(1)}\left(x_{\mathrm{a}}, y_{\mathrm{b}}, y_{\mathrm{r}}, \hat{y}_{\mathbf{r}} \mid q\right) p^{(2)}\left(x_{\mathrm{b}}, y_{\mathrm{a}}, y_{\mathrm{r}}, \hat{y}_{\mathrm{r}} \mid q\right) p^{(3)}\left(u_{\mathrm{a}}, u_{\mathrm{b}}, x_{\mathrm{r}}, y_{\mathrm{a}}, y_{\mathrm{b}} \mid q\right) p^{(4)}\left(x_{\mathrm{r}}, y_{\mathrm{a}}, y_{\mathrm{b}}\right)
\end{aligned}
$$

where

$$
\begin{aligned}
p^{(1)}\left(x_{\mathrm{a}}, y_{\mathrm{b}}, y_{\mathrm{r}}, \hat{y}_{\mathrm{r}} \mid q\right) & =p^{(1)}\left(x_{\mathrm{a}} \mid q\right) p^{(1)}\left(y_{\mathrm{b}}, y_{\mathrm{r}} \mid x_{\mathrm{a}}\right) p^{(1)}\left(\hat{y}_{\mathrm{r}} \mid y_{\mathrm{r}}, q\right) \\
p^{(2)}\left(x_{\mathrm{b}}, y_{\mathrm{a}}, y_{\mathrm{r}}, \hat{y}_{\mathrm{r}} \mid q\right) & =p^{(2)}\left(x_{\mathrm{b}} \mid q\right) p^{(2)}\left(y_{\mathrm{a}}, y_{\mathrm{r}} \mid x_{\mathrm{b}}\right) p^{(2)}\left(\hat{y}_{\mathrm{r}} \mid y_{\mathrm{r}}, q\right) \\
p^{(3)}\left(u_{\mathrm{a}}, u_{\mathrm{b}}, x_{\mathrm{r}}, y_{\mathrm{a}}, y_{\mathrm{b}} \mid q\right) & =p^{(3)}\left(u_{\mathrm{a}}, u_{\mathrm{b}} \mid q\right) p^{(3)}\left(x_{\mathrm{r}} \mid u_{\mathrm{a}}, u_{\mathrm{b}}, q\right) p^{(3)}\left(y_{\mathrm{a}}, y_{\mathrm{b}} \mid x_{\mathrm{r}}\right) \\
p^{(4)}\left(x_{\mathrm{r}}, y_{\mathrm{a}}, y_{\mathrm{b}}\right) & =p^{(4)}\left(x_{\mathrm{r}}\right) p^{(4)}\left(y_{\mathrm{a}}, y_{\mathrm{b}} \mid x_{\mathrm{r}}\right)
\end{aligned}
$$

with $|\mathcal{Q}| \leq 13,\left|\hat{\mathcal{Y}}_{\mathrm{r}}\right| \leq|\mathcal{Q}|\left|\mathcal{Y}_{\mathrm{r}}\right|+3$ over the alphabet $\mathcal{X}_{\mathrm{a}} \times \mathcal{X}_{\mathrm{b}} \times \mathcal{X}_{\mathrm{r}}^{2} \times \mathcal{Y}_{\mathrm{a}}^{3} \times \mathcal{Y}_{\mathrm{b}}^{3} \times \mathcal{Y}_{\mathrm{r}}^{2} \times \hat{\mathcal{Y}}_{\mathrm{r}}^{2}$.

Remark 1: We note that the division of the relay broadcast phase into two sub-phases allows for a simple reduction of the protocol to known transmission schemes. In particular, if the direct-links $a \rightarrow b$ and 
$\mathrm{b} \rightarrow \mathrm{a}$ are very weak, i.e., $I\left(\hat{Y}_{\mathrm{r}}^{(1)} ; Y_{\mathrm{b}}^{(1)}\right)$ and $I\left(\hat{Y}_{\mathrm{r}}^{(2)} ; Y_{\mathrm{b}}^{(2)}\right)$ are very small and the users' own messages are of limited use to cancel out interference in phase 4, i.e., $I\left(Y_{\mathrm{r}}^{(1)} ; \hat{Y}_{\mathrm{r}}^{(1)} \mid X_{\mathrm{a}}^{(1)}\right) \approx I\left(Y_{\mathrm{r}}^{(1)} ; \hat{Y}_{\mathrm{r}}^{(1)}\right)$ and $I\left(Y_{\mathrm{r}}^{(2)} ; \hat{Y}_{\mathrm{r}}^{(2)} \mid X_{\mathrm{b}}^{(2)}\right) \approx I\left(Y_{\mathrm{r}}^{(2)} ; \hat{Y}_{\mathrm{r}}^{(2)}\right)$ a classical broadcast channel may be more beneficial and we may let $\Delta_{4} \rightarrow 0, \alpha_{\mathrm{a}}, \alpha_{\mathrm{b}} \rightarrow 1$ such that the relay phase corresponds to a classical broadcast channel. At the opposite extreme, when the direct links provide a large amount of side-information, i.e., $I\left(\hat{Y}_{\mathrm{r}}^{(1)} ; Y_{\mathrm{b}}^{(1)}\right)$ and $I\left(\hat{Y}_{\mathrm{r}}^{(2)} ; Y_{\mathrm{b}}^{(2)}\right)$ are large and the users' own message knowledge may be used to cancel out almost all "interference" in a broadcast scheme in which a common message is sent, i.e., $I\left(Y_{\mathrm{r}}^{(1)} ; \hat{Y}_{\mathrm{r}}^{(1)} \mid X_{\mathrm{a}}^{(1)}\right)$ and $I\left(Y_{\mathrm{r}}^{(2)} ; \hat{Y}_{\mathrm{r}}^{(2)} \mid X_{\mathrm{b}}^{(2)}\right)$ are very small, we may set $\Delta_{3} \rightarrow 0, \alpha_{\mathrm{a}}, \alpha_{\mathrm{b}} \rightarrow 0 .{ }^{2}$ The detailed proof is provided in Appendix A.

Remark 2: Strong typicality is required for the proof of Theorem 1 in order to apply the Markov lemma to $\left(X_{\mathrm{a}}^{(1)}, X_{\mathrm{b}}^{(1)}\right) \rightarrow Y_{\mathrm{r}}^{(1)} \rightarrow \hat{Y}_{\mathrm{r}}^{(1)}$ for each given $q$. Since strong typicality is defined for discrete alphabets, Theorem 1 cannot be directly extended to continuous alphabets. However, the extended Markov lemma (see Remark 30 of [14] as well as Lemma 3 of [23]) shows that for Gaussian distributions, the Markov lemma still applies.

Remark 3: We use coded time sharing [10] so that (3)-(7) will hold for a larger set of distributions than for regular time-sharing. For regular time-sharing, these equations would need to hold for all individual distributions, that is, $\alpha_{\mathrm{a}} \Delta_{1} I\left(Y_{\mathrm{r}}^{(1)} ; \hat{Y}_{\mathrm{r}}^{(1)}\right)<\Delta_{3} I\left(U_{\mathrm{a}}^{(3)} ; Y_{\mathrm{b}}^{(3)}\right)$ for all $p^{(1)}\left(y_{\mathrm{r}}, \hat{y}_{\mathrm{r}}\right)$ in (3). However, with coded time sharing, we only need these constraints to hold for the convex combination of the individual mutual information terms, that is, $\alpha_{\mathrm{a}} \Delta_{1} I\left(Y_{\mathrm{r}}^{(1)} ; \hat{Y}_{\mathrm{r}}^{(1)} \mid Q\right)<\Delta_{3} I\left(U_{\mathrm{a}}^{(3)} ; Y_{\mathrm{b}}^{(3)}\right)$ for all $p^{(1)}\left(y_{\mathrm{r}}, \hat{y}_{\mathrm{r}}\right)$, yielding a larger set of distributions over which the rate region is taken and therefore a possibly larger achievable rate region.

When the direct forward and reverse links are of different strength, a scheme in which one direction uses a CF and the other uses a DF relaying scheme may provide a larger rate region than if both links use CF. In the next theorem, we provide a rate region for a TDBC scenario in which the forward link uses DF and the reverse link uses CF.

Theorem 2: An achievable rate region for the half-duplex bi-directional relay channel with a mixed TDBC protocol, where the $\mathrm{a} \rightarrow \mathrm{r} \rightarrow \mathrm{b}$ link uses decode and forward and the $\mathrm{a} \leftarrow \mathrm{r} \leftarrow \mathrm{b}$ link uses compress and forward, is the closure of the set of all points $\left(R_{\mathrm{a}}, R_{\mathrm{b}}\right)$ satisfying

$$
R_{\mathrm{a}}<\min \left\{\Delta_{1} I\left(X_{\mathrm{a}}^{(1)} ; Y_{\mathrm{r}}^{(1)}\right), \Delta_{1} I\left(X_{\mathrm{a}}^{(1)} ; Y_{\mathrm{b}}^{(1)}\right)+\Delta_{3} I\left(U_{\mathrm{r}}^{(3)} ; Y_{\mathrm{b}}^{(3)} \mid Q\right)-\Delta_{3} I\left(U_{\mathrm{r}}^{(3)} ; U_{\mathrm{b}}^{(3)} \mid Q\right)\right\}
$$

${ }^{2}$ This choice of $\Delta_{3}, \alpha_{\mathrm{a}}, \alpha_{\mathrm{b}}$ is on the boundary of the closure of the achievable rate region. 


$$
R_{\mathrm{b}}<\Delta_{2} I\left(X_{\mathrm{b}}^{(2)} ; \hat{Y}_{\mathrm{r}}^{(2)}, Y_{\mathrm{a}}^{(2)} \mid Q\right)
$$

subject to

$$
\Delta_{2} I\left(Y_{\mathrm{r}}^{(2)} ; \hat{Y}_{\mathrm{r}}^{(2)} \mid Y_{\mathrm{a}}^{(2)}, Q\right)<\min \left\{\Delta_{3} I\left(U_{\mathrm{r}}^{(3)}, U_{\mathrm{b}}^{(3)} ; Y_{\mathrm{a}}^{(3)} \mid Q\right), \Delta_{3} I\left(U_{\mathrm{b}}^{(3)} ; U_{\mathrm{r}}^{(3)}, Y_{\mathrm{a}}^{(3)} \mid Q\right)\right\}
$$

over all joint distributions,

$$
p\left(q, x_{\mathrm{a}}, x_{\mathrm{b}}, x_{\mathrm{r}}, u_{\mathrm{b}}, u_{\mathrm{r}}, y_{\mathrm{a}}, y_{\mathrm{b}}, y_{\mathrm{r}}, \hat{y}_{\mathrm{r}}\right)=p(q) p^{(1)}\left(x_{\mathrm{a}}, y_{\mathrm{b}}, y_{\mathrm{r}}\right) p^{(2)}\left(x_{\mathrm{b}}, y_{\mathrm{a}}, y_{\mathrm{r}}, \hat{y}_{\mathrm{r}} \mid q\right) p^{(3)}\left(u_{\mathrm{b}}, u_{\mathrm{r}}, x_{\mathrm{r}}, y_{\mathrm{a}}, y_{\mathrm{b}} \mid q\right)
$$

where

$$
\begin{gathered}
p^{(1)}\left(x_{\mathrm{a}}, y_{\mathrm{b}}, y_{\mathrm{r}}\right)=p^{(1)}\left(x_{\mathrm{a}}\right) p^{(1)}\left(y_{\mathrm{b}}, y_{\mathrm{r}} \mid x_{\mathrm{a}}\right) \\
p^{(2)}\left(x_{\mathrm{b}}, y_{\mathrm{a}}, y_{\mathrm{r}}, \hat{y}_{\mathrm{r}} \mid q\right)=p^{(2)}\left(x_{\mathrm{b}} \mid q\right) p^{(2)}\left(y_{\mathrm{a}}, y_{\mathrm{r}} \mid x_{\mathrm{b}}\right) p^{(2)}\left(\hat{y}_{\mathrm{r}} \mid y_{\mathrm{r}}, q\right) \\
p^{(3)}\left(u_{\mathrm{a}}, u_{\mathrm{b}}, u_{\mathrm{r}}, x_{\mathrm{r}}, y_{\mathrm{a}}, y_{\mathrm{b}} \mid q\right)=p^{(3)}\left(u_{\mathrm{b}}, u_{\mathrm{r}} \mid q\right) p^{(3)}\left(x_{\mathrm{r}} \mid u_{\mathrm{b}}, u_{\mathrm{r}}, q\right) p^{(3)}\left(y_{\mathrm{a}}, y_{\mathrm{b}} \mid x_{\mathrm{r}}\right)
\end{gathered}
$$

with $|\mathcal{Q}| \leq 6,\left|\hat{\mathcal{Y}}_{\mathrm{r}}\right| \leq|\mathcal{Q}|\left|\mathcal{Y}_{\mathrm{r}}\right|+2$ over the alphabet $\mathcal{X}_{\mathrm{a}} \times \mathcal{X}_{\mathrm{b}} \times \mathcal{X}_{\mathrm{r}} \times \mathcal{U}_{\mathrm{b}} \times \mathcal{U}_{\mathrm{r}} \times \mathcal{Y}_{\mathrm{a}}^{2} \times \mathcal{Y}_{\mathrm{b}}^{2} \times \mathcal{Y}_{\mathrm{r}}^{2} \times \hat{\mathcal{Y}}_{\mathrm{r}}$

Proof outline : We use random (Slepian-Wolf-like) binning to exploit the overheard side information and a Gel'fand-Pinsker coding scheme to broadcast two separate messages from the relay to the terminal nodes. Theorem 2 then follows the same argument as the proof of Theorem 1. We note that the mixed TDBC protocol, the DF TDBC and the CF TDBC protocol are not generally ordered in terms of performance, i.e., one can find channel scenarios in which each one achieves "better" rates than the others.

\section{B. MABC Protocol}

During phase 1 , nodes $\mathrm{a}$ and $\mathrm{b}$ simultaneously send independent messages $w_{\mathrm{a}}$ and $w_{\mathrm{b}}$ as codewords $\mathbf{x}_{\mathrm{a}}^{(1)}\left(w_{\mathrm{a}}\right)$ and $\mathbf{x}_{\mathrm{b}}^{(1)}\left(w_{\mathrm{b}}\right)$ to the relay, forming a classical multiple-access channel. Since we assume halfduplex nodes, neither a nor $b$ can receive the message of the other during phase 1 and hence no directlink side information is available at the terminal nodes. The relay receives the signal $\mathbf{y}_{\mathbf{r}}^{(1)}$ according to $p^{(1)}\left(y_{\mathrm{r}} \mid x_{\mathrm{a}}, x_{\mathrm{b}}\right)$. Rather than attempting to decode message $w_{\mathrm{a}}$ and $w_{\mathrm{b}}$ (as in a DF scheme), it compresses the received $\mathbf{y}_{\mathbf{r}}^{(1)}$ into a signal $\hat{\mathbf{y}}_{r}^{(1)}\left(w_{\mathbf{r}}\right)$. The index $w_{\mathrm{r}}$ is then mapped in a one-to-one fashion to the codeword $\mathbf{x}_{r}^{(2)}\left(w_{r}\right)$ which is broadcast in phase 2 back to the relays. The challenge here is to determine the optimal compression strategy such that just enough information is carried back to the nodes to decode the opposite node's message, by fully exploiting the own-message side-information available at each terminal. 
Theorem 3: An achievable rate region of the half-duplex bi-directional relay channel with the compress and forward MABC protocol is the closure of the set of all points $\left(R_{\mathrm{a}}, R_{\mathrm{b}}\right)$ satisfying

$$
\begin{aligned}
& R_{\mathrm{a}}<\Delta_{1} I\left(X_{\mathrm{a}}^{(1)} ; \hat{Y}_{\mathrm{r}}^{(1)} \mid X_{\mathrm{b}}^{(1)}, Q\right) \\
& R_{\mathrm{b}}<\Delta_{1} I\left(X_{\mathrm{b}}^{(1)} ; \hat{Y}_{\mathrm{r}}^{(1)} \mid X_{\mathrm{a}}^{(1)}, Q\right)
\end{aligned}
$$

subject to

$$
\begin{aligned}
& \Delta_{1} I\left(Y_{\mathrm{r}}^{(1)} ; \hat{Y}_{\mathrm{r}}^{(1)} \mid X_{\mathrm{b}}^{(1)}, Q\right)<\Delta_{2} I\left(X_{\mathrm{r}}^{(2)} ; Y_{\mathrm{b}}^{(2)}\right) \\
& \Delta_{1} I\left(Y_{\mathrm{r}}^{(1)} ; \hat{Y}_{\mathrm{r}}^{(1)} \mid X_{\mathrm{a}}^{(1)}, Q\right)<\Delta_{2} I\left(X_{\mathrm{r}}^{(2)} ; Y_{\mathrm{a}}^{(2)}\right)
\end{aligned}
$$

over all joint distributions,

$$
p\left(q, x_{\mathrm{a}}, x_{\mathrm{b}}, x_{\mathrm{r}}, y_{\mathrm{a}}, y_{\mathrm{b}}, y_{\mathrm{r}}, \hat{y}_{\mathrm{r}}\right)=p^{(1)}\left(q, x_{\mathrm{a}}, x_{\mathrm{b}}, y_{\mathrm{r}}, \hat{y}_{\mathrm{r}}\right) p^{(2)}\left(x_{\mathrm{r}}, y_{\mathrm{a}}, y_{\mathrm{b}}\right)
$$

where

$$
\begin{aligned}
p^{(1)}\left(q, x_{\mathrm{a}}, x_{\mathrm{b}}, y_{\mathrm{r}}, \hat{y}_{\mathrm{r}}\right) & =p^{(1)}(q) p^{(1)}\left(x_{\mathrm{a}} \mid q\right) p^{(1)}\left(x_{\mathrm{b}} \mid q\right) p^{(1)}\left(y_{\mathrm{r}} \mid x_{\mathrm{a}}, x_{\mathrm{b}}\right) p^{(1)}\left(\hat{y}_{\mathrm{r}} \mid y_{\mathrm{r}}, q\right) \\
p^{(2)}\left(x_{\mathrm{r}}, y_{\mathrm{a}}, y_{\mathrm{b}}\right) & =p^{(2)}\left(x_{\mathrm{r}}\right) p^{(2)}\left(y_{\mathrm{a}}, y_{\mathrm{b}} \mid x_{\mathrm{r}}\right)
\end{aligned}
$$

with $|\mathcal{Q}| \leq 4,\left|\hat{\mathcal{Y}}_{\mathrm{r}}\right| \leq|\mathcal{Q}|\left|\mathcal{Y}_{\mathrm{r}}\right|+4$ over the alphabet $\mathcal{X}_{\mathrm{a}} \times \mathcal{X}_{\mathrm{b}} \times \mathcal{X}_{\mathrm{r}} \times \mathcal{Y}_{\mathrm{a}} \times \mathcal{Y}_{\mathrm{b}} \times \mathcal{Y}_{\mathrm{r}} \times \hat{\mathcal{Y}}_{\mathrm{r}}$

Remark 4: The bound of Theorem 3 is essentially independently derived in [27]. We do however note that equation (25) is a slight extension of the work in [27], as we use $p^{(1)}\left(\hat{y}_{\mathbf{r}} \mid y_{\mathbf{r}}, q\right)$ instead of $p^{(1)}\left(\hat{y}_{\mathbf{r}} \mid y_{\mathbf{r}}\right)$, i.e., in [27] the compression codewords $\hat{\mathbf{y}}_{\mathbf{r}}^{(1)}$ are generated according to $p^{(1)}\left(\hat{y}_{\mathbf{r}}\right)=\sum p^{(1)}\left(y_{\mathbf{r}}\right) p^{(1)}\left(\hat{y}_{\mathbf{r}} \mid y_{\mathbf{r}}\right)$, while in (25) the space of compression distributions $p^{(1)}\left(\hat{y}_{\mathbf{r}} \mid q\right)=\sum p^{(1)}\left(y_{\mathbf{r}} \mid q\right) p^{(1)}\left(\hat{y}_{\mathbf{r}} \mid y_{\mathbf{r}}, q\right)$ is in general larger. By conditioning on $q$, one can "fine-tune" the distribution of $\hat{y}_{\mathrm{r}}^{(1)}$ for each given $q$ and the left side of (22) and (23) may be reduced. This is because the distributions of $X_{\mathrm{a}}^{(1)}$ and $X_{\mathrm{b}}^{(1)}$, and hence $Y_{\mathrm{r}}^{(1)}$, depend on $q$. For example, let $p^{(1)}(q=1)=\alpha_{n}$ and $p^{(1)}(q=2)=1-\alpha_{n}$, where $0<\alpha_{n}<$ 1. For $q=1$ we optimize $p^{(1)}\left(\hat{y}_{\mathrm{r}} \mid 1\right)$ and generate $\left(\alpha_{n} \Delta_{1, n} \cdot n\right)$-length sequence $\hat{\mathbf{y}}_{\mathrm{r}}^{(1), 1}\left(w_{\mathrm{r} 1}\right), w_{\mathrm{r} 1} \in$ $\left\{0,1, \cdots\left\lfloor 2^{n R_{\mathrm{r} 1}}\right\rfloor\right\}$, where $R_{\mathrm{r} 1}=\alpha_{n} \Delta_{1, n}\left(I\left(Y_{\mathrm{r}}^{(1)} ; \hat{Y}_{\mathrm{r}}^{(1)} \mid q=1\right)+\epsilon\right)$. Likewise, we generate $\hat{\mathbf{y}}_{\mathrm{r}}^{(1), 2}\left(w_{\mathrm{r} 2}\right)$ for $q=2$. To compress $\mathbf{y}_{\mathbf{r}}^{(1)}$ to $\hat{\mathbf{y}}_{\mathbf{r}}^{(1)}$, we construct $\mathbf{y}_{\mathbf{r}}^{(1)}=\left(\mathbf{y}_{\mathbf{r}}^{(1), 1}, \mathbf{y}_{\mathbf{r}}^{(1), 2}\right)$ and $\hat{\mathbf{y}}_{\mathbf{r}}^{(1)}=\left(\hat{\mathbf{y}}_{\mathbf{r}}^{(1), 1}, \hat{\mathbf{y}}_{\mathbf{r}}^{(1), 2}\right)$ and choose $w_{\mathbf{r}}=\left(w_{\mathrm{r} 1}, w_{\mathbf{r} 2}\right)$ if both $\left(\mathbf{y}_{\mathbf{r}}^{(1), 1}, \hat{\mathbf{y}}_{\mathbf{r}}^{(1), 1}\left(w_{\mathrm{r} 1}\right)\right)$ and $\left(\mathbf{y}_{\mathbf{r}}^{(1), 2}, \hat{\mathbf{y}}_{\mathbf{r}}^{(1), 2}\left(w_{\mathrm{r} 2}\right)\right)$ are jointly typical. Then the rate $R_{\mathrm{r}}=R_{\mathrm{r} 1}+R_{\mathrm{r} 2}=\Delta_{1} I\left(Y_{\mathrm{r}}^{(1)} ; \hat{Y}_{\mathrm{r}}^{(1)} \mid Q\right)$. However, if one generated $\hat{\mathbf{y}}_{\mathrm{r}}^{(1)}$ from the distribution $p^{(1)}\left(\hat{y}_{\mathrm{r}}\right)$ then $R_{\mathrm{r}}=\Delta_{1} I\left(Y_{\mathrm{r}}^{(1)} ; \hat{Y}_{\mathrm{r}}^{(1)}\right)$ which in general could be either smaller or greater than $\Delta_{1} I\left(Y_{\mathrm{r}}^{(1)} ; \hat{Y}_{\mathrm{r}}^{(1)} \mid Q\right)$ depending on the particular $p^{(1)}\left(y_{\mathrm{r}} \mid q\right)$ and $p^{(1)}\left(\hat{y}_{\mathrm{r}} \mid y_{\mathrm{r}}, q\right)$, but for optimized $p^{(1)}\left(\hat{y}_{\mathrm{r}} \mid y_{\mathrm{r}}, q\right)$ is always smaller or equal with equality only in degenerate cases. A similar argument may be found in [7]. 
In the TDBC protocol, we provided achievable rate regions for both $\mathrm{CF}$ and Mixed ( $\mathrm{CF}$ one way, DF the other) forwarding schemes. In general, no strict relationship between the CF, DF and Mixed forwarding schemes exists for the TDBC protocol. In the MABC protocol, the $\mathrm{CF}$ and DF forwarding schemes will be shown numerically to not contain each other in two-way AWGN channels. We do not present a Mixed MABC region in which $\mathrm{CF}$ is used in one direction and $\mathrm{DF}$ in the other as in such a scheme 1$)$ one message $\left(w_{\mathrm{a}}\right)$ still has to be decoded at the relay, and 2$)$ the compressed signal $\left(\hat{y}_{\mathrm{r}}\right)$ contains less information than the received signal $\left(y_{r}\right)$. Using these, one may show that a MABC protocol with a Mixed forwarding scheme which uses techniques similar to those used in the Mixed TDBC protocol is completely included in the DF MABC protocol. As such, the Mixed MABC region is omitted.

\section{Gaussian CASE}

We now assume all links in the bi-directional relay channel are subject to independent, identically distributed white Gaussian noise. The commonly considered Gaussian channel will allow us to visually compare different achievable rate regions for the bi-directional relaying channel. Definitions of codes, rate, and achievability in the memoryless Gaussian channels are analogous to those of the discrete memoryless channels. The achievable rate regions for the Gaussian noise channel are obtained by evaluation of the previously derived rate regions for Gaussian input distributions. We note that since strong typicality needed for the CF forwarding schemes - does not apply to continuous random variables, the achievable rate regions from the theorems in the previous section do not directly apply to continuous domains. However, for the Gaussian input distributions and additive Gaussian noise which we will assume in the following, the Markov lemma of [23], which generalizes the Markov lemma to the continuous domains, ensures that the achievable rate regions in the previous section are valid for AWGN channels. The corresponding Gaussian channel model is:

$$
\begin{aligned}
& Y_{\mathrm{a}}[m]=h_{\mathrm{ra}} X_{\mathrm{r}}[m]+h_{\mathrm{ba}} X_{\mathrm{b}}[m]+Z_{\mathrm{a}}[m] \\
& Y_{\mathrm{b}}[m]=h_{\mathrm{rb}} X_{\mathrm{r}}[m]+h_{\mathrm{ab}} X_{\mathrm{a}}[m]+Z_{\mathrm{b}}[m] \\
& Y_{\mathrm{r}}[m]=h_{\mathrm{ar}} X_{\mathrm{a}}[m]+h_{\mathrm{br}} X_{\mathrm{b}}[m]+Z_{\mathrm{r}}[m]
\end{aligned}
$$

where $X_{\mathrm{a}}[m], X_{\mathrm{b}}[m]$ and $X_{\mathrm{r}}[m]$ follow the input distributions $X_{\mathrm{a}}^{(\ell)} \sim \mathcal{C N}\left(0, P_{\mathrm{a}}\right), X_{\mathrm{b}}^{(\ell)} \sim \mathcal{C N}\left(0, P_{\mathrm{b}}\right)$ and $X_{\mathrm{r}}^{(\ell)} \sim \mathcal{C N}\left(0, P_{\mathrm{r}}\right), m \in\left[n \sum_{j=0}^{\ell-1} \Delta_{j, n}+1, n \sum_{j=0}^{\ell} \Delta_{j, n}\right]$, and $\mathcal{C N}\left(\mu, \sigma^{2}\right)$ denotes a complex Gaussian random variable with mean $\mu$ and variance $\sigma^{2}$, and $\ell$ corresponds to the appropriate phase. If node $i$ is transmitting, its transmit power is bounded by $P_{i}$, i.e., $E\left[\left|X_{i}\right|^{2}\right] \leq P_{i}$. If node $i$ is receiving, its input 
symbol during that phase does not exist in the above mathematical channel model. For example, in the first phase of the TDBC protocol, the corresponding channel model is :

$$
\begin{aligned}
& Y_{\mathrm{b}}[m]=h_{\mathrm{ab}} X_{\mathrm{a}}[m]+Z_{\mathrm{b}}[m] \\
& Y_{\mathrm{r}}[m]=h_{\mathrm{ar}} X_{\mathrm{a}}[m]+Z_{\mathrm{r}}[m] .
\end{aligned}
$$

In the above $h_{i j}(\in \mathbb{C})$ is the effective channel gain between transmitter $i$ and receiver $j$. We assume that the channel is reciprocal such that $h_{i j}=h_{j i}$ and each node is fully aware of $h_{\mathrm{ar}}, h_{\mathrm{br}}$ and $h_{\mathrm{ab}}$ (i.e. full CSI). The noise at all receivers $Z_{\mathrm{a}}, Z_{\mathrm{b}}, Z_{\mathrm{r}}$ is of unit power, additive, white Gaussian, complex and circularly symmetric. For convenience of analysis, we also define the function $C(x):=\log _{2}(1+x)$. For the analysis of the Compress and Forward scheme, we assume $\hat{Y}_{\mathrm{r}}^{(\ell)}$ are zero mean Gaussians and define $P_{y}^{(\ell)}:=E\left[\left|Y_{r}^{(\ell)}\right|^{2}\right], P_{\hat{y}}^{(\ell)}:=E\left[\left|\hat{Y}_{r}^{(\ell)}\right|^{2}\right]$ and $\sigma_{y}^{(\ell)}:=E\left[\left|\hat{Y}_{r}^{(\ell)} Y_{r}^{(\ell)}\right|\right]$. Then the relation between the received $Y_{\mathrm{r}}[m]$ and the compressed $\hat{Y}_{\mathrm{r}}[m]$ are given by the following equivalent channel model:

$$
\hat{Y}_{\mathrm{r}}[m]=h_{\mathrm{r} \hat{r}}[m] Y_{\mathrm{r}}[m]+Z_{\hat{\mathrm{r}}}[m],
$$

where $Y_{\mathrm{r}}[m], \hat{Y}_{\mathrm{r}}[m]$ and $Z_{\hat{\mathrm{r}}}[m]$ follow the distributions $Y_{\mathrm{r}}^{(\ell)} \sim \mathcal{C N}\left(0, P_{y}^{(\ell)}\right), \hat{Y}_{\mathrm{r}}^{(\ell)} \sim \mathcal{C N}\left(0, P_{\hat{y}}^{(\ell)}\right)$ and $Z_{\hat{\mathrm{r}}}^{(\ell)} \sim \mathcal{C N}\left(0, P_{\hat{y}}^{(\ell)}-\frac{\left(\sigma_{y}^{(\ell)}\right)^{2}}{P_{y}^{(\ell)}}\right)$ and $h_{\mathrm{r} \hat{\mathrm{r}}}[m]=\frac{\sigma_{y}^{(\ell)}}{P_{y}^{(\ell)}}$, where $m \in\left[n \sum_{j=0}^{\ell-1} \Delta_{j, n}+1, n \sum_{j=0}^{\ell} \Delta_{j, n}\right]$. We note that in the following, $P_{\hat{y}}^{(\ell)}$ and $\sigma_{y}^{(\ell)}$ are variables corresponding to the compression that are numerically optimized. We consider five different relaying schemes for the MABC and TDBC bi-directional protocols: Amplify and Forward (AF), Decode and Forward (DF), Lattice Forwarding (Lattice), Compress and Forward (CF), and Mixed Forward (Mixed). In addition to achievable rate regions, we apply outer bounds for the MABC and TDBC protocols to the Gaussian channel.

\section{A. Amplify and Forward}

In the amplify and forward scheme, all phase durations are equal, since relaying is performed on a symbol by symbol basis. Therefore, $\Delta_{1}=\Delta_{2}=\frac{1}{2}$ for the MABC protocol and $\Delta_{1}=\Delta_{2}=\Delta_{3}=\frac{1}{3}$ for the TDBD protocol. Furthermore, relay $r$ scales the received symbol $y_{r}$ by $\sqrt{\frac{P_{r}}{P_{y}}}$ to meet the transmit power constraint of $P_{\mathrm{r}}$. The following are achievable rate regions for the amplify and forward relaying:

- MABC Protocol

$$
\begin{aligned}
& R_{\mathrm{a}}<\frac{1}{2} C\left(\frac{\left|h_{\mathrm{ar}}\right|^{2}\left|h_{\mathrm{br}}\right|^{2} P_{\mathrm{a}} P_{\mathrm{r}}}{\left|h_{\mathrm{ar}}\right|^{2} P_{\mathrm{a}}+\left|h_{\mathrm{br}}\right|^{2} P_{\mathrm{b}}+\left|h_{\mathrm{br}}\right|^{2} P_{\mathrm{r}}+1}\right) \\
& R_{\mathrm{b}}<\frac{1}{2} C\left(\frac{\left|h_{\mathrm{ar}}\right|^{2}\left|h_{\mathrm{br}}\right|^{2} P_{\mathrm{b}} P_{\mathrm{r}}}{\left|h_{\mathrm{ar}}\right|^{2} P_{\mathrm{a}}+\left|h_{\mathrm{br}}\right|^{2} P_{\mathrm{b}}+\left|h_{\mathrm{ar}}\right|^{2} P_{\mathrm{r}}+1}\right)
\end{aligned}
$$


- TDBC Protocol

$$
\begin{aligned}
R_{\mathrm{a}} & <\frac{1}{3} C\left(\left|h_{\mathrm{ab}}\right|^{2} P_{\mathrm{a}}+\frac{\left|h_{\mathrm{ar}}\right|^{2}\left|h_{\mathrm{br}}\right|^{2} P_{\mathrm{a}} P_{\mathrm{r}}}{\left|h_{\mathrm{ar}}\right|^{2} P_{\mathrm{a}}+\left|h_{\mathrm{br}}\right|^{2} P_{\mathrm{b}}+2\left|h_{\mathrm{br}}\right|^{2} P_{\mathrm{r}}+2}\right) \\
R_{\mathrm{b}} & <\frac{1}{3} C\left(\left|h_{\mathrm{ab}}\right|^{2} P_{\mathrm{b}}+\frac{\left|h_{\mathrm{ar}}\right|^{2}\left|h_{\mathrm{br}}\right|^{2} P_{\mathrm{b}} P_{\mathrm{r}}}{\left|h_{\mathrm{ar}}\right|^{2} P_{\mathrm{a}}+\left|h_{\mathrm{br}}\right|^{2} P_{\mathrm{b}}+2\left|h_{\mathrm{ar}}\right|^{2} P_{\mathrm{r}}+2}\right)
\end{aligned}
$$

\section{B. Decode and Forward}

Applying Theorems 2 and 3 in [13] to the Gaussian case, we obtain the following achievable rate regions:

- MABC Protocol

$$
\begin{array}{r}
R_{\mathrm{a}}<\min \left\{\Delta_{1} C\left(\left|h_{\mathrm{ar}}\right|^{2} P_{\mathrm{a}}\right), \Delta_{2} C\left(\left|h_{\mathrm{br}}\right|^{2} P_{\mathrm{r}}\right)\right\} \\
R_{\mathrm{b}}<\min \left\{\Delta_{1} C\left(\left|h_{\mathrm{br}}\right|^{2} P_{\mathrm{b}}\right), \Delta_{2} C\left(\left|h_{\mathrm{ar}}\right|^{2} P_{\mathrm{r}}\right)\right\} \\
R_{\mathrm{a}}+R_{\mathrm{b}}<\Delta_{1} C\left(\left|h_{\mathrm{ar}}\right|^{2} P_{\mathrm{a}}+\left|h_{\mathrm{br}}\right|^{2} P_{\mathrm{b}}\right)
\end{array}
$$

- TDBC Protocol

$$
\begin{aligned}
& R_{\mathrm{a}}<\min \left\{\Delta_{1} C\left(\left|h_{\mathrm{ar}}\right|^{2} P_{\mathrm{a}}\right), \Delta_{1} C\left(\left|h_{\mathrm{ab}}\right|^{2} P_{\mathrm{a}}\right)+\Delta_{3} C\left(\left|h_{\mathrm{br}}\right|^{2} P_{\mathrm{r}}\right)\right\} \\
& R_{\mathrm{b}}<\min \left\{\Delta_{2} C\left(\left|h_{\mathrm{br}}\right|^{2} P_{\mathrm{b}}\right), \Delta_{2} C\left(\left|h_{\mathrm{ab}}\right|^{2} P_{\mathrm{b}}\right)+\Delta_{3} C\left(\left|h_{\mathrm{ar}}\right|^{2} P_{\mathrm{r}}\right)\right\}
\end{aligned}
$$

When obtaining the regions numerically, we optimize $\Delta_{\ell}$ 's for the given channel mutual informations to maximize the achievable rate regions.

\section{Compress and Forward}

Applying Theorem 3 and 1 to the Gaussian case, we obtain the following achievable rate regions:

- MABC Protocol

$$
\begin{aligned}
& R_{\mathrm{a}}<\Delta_{1} C\left(\frac{\left(\sigma_{y}^{(1)}\right)^{2}\left|h_{\mathrm{ar}}\right|^{2} P_{\mathrm{a}}}{P_{\hat{y}}^{(1)}\left(P_{y}^{(1)}\right)^{2}-\left(\sigma_{y}^{(1)}\right)^{2}\left(P_{y}^{(1)}-1\right)}\right) \\
& R_{\mathrm{b}}<\Delta_{1} C\left(\frac{\left(\sigma_{y}^{(1)}\right)^{2}\left|h_{\mathrm{br}}\right|^{2} P_{\mathrm{b}}}{P_{\hat{y}}^{(1)}\left(P_{y}^{(1)}\right)^{2}-\left(\sigma_{y}^{(1)}\right)^{2}\left(P_{y}^{(1)}-1\right)}\right)
\end{aligned}
$$

where,

$$
\begin{aligned}
& \Delta_{1}=\min \left\{\frac{C\left(\left|h_{\mathrm{br}}\right|^{2} P_{\mathrm{r}}\right)}{C\left(\frac{\left(\sigma_{y}^{(1)}\right)^{2}\left(\left|h_{\mathrm{ar}}\right|^{2} P_{\mathrm{a}}+1\right)}{P_{\hat{y}}^{(1)}\left(P_{y}^{(1)}\right)^{2}-\left(\sigma_{y}^{(1)}\right)^{2} P_{y}^{(1)}}\right)+C\left(\left|h_{\mathrm{br}}\right|^{2} P_{\mathrm{r}}\right)}, \frac{C\left(\left|h_{\mathrm{ar}}\right|^{2} P_{\mathrm{r}}\right)}{C\left(\frac{\left(\sigma_{y}^{(1)}\right)^{2}\left(\left|h_{\mathrm{br}}\right|^{2} P_{\mathrm{b}}+1\right)}{P_{\grave{y}}^{(1)}\left(P_{y}^{(1)}\right)^{2}-\left(\sigma_{y}^{(1)}\right)^{2} P_{y}^{(1)}}\right)+C\left(\left|h_{\mathrm{ar}}\right|^{2} P_{\mathrm{r}}\right)}\right\} \\
& P_{y}^{(1)}=\left|h_{\mathrm{ar}}\right|^{2} P_{\mathrm{a}}+\left|h_{\mathrm{br}}\right|^{2} P_{\mathrm{b}}+1 .
\end{aligned}
$$


- TDBC Protocol One can show that Marton's bound in (3) - (5) is equivalent to the capacity region of the Gaussian broadcast channel with Costa's setup as follows: let $\left|h_{\mathrm{ra}}\right|>\left|h_{\mathrm{rb}}\right|$ and we set

$$
\text { In phase } 3\left\{\begin{array}{l}
U_{\mathrm{b}}[m]=V_{\mathrm{r}}[m]+\alpha U_{\mathrm{a}}[m] \\
Y_{\mathrm{a}}[m]=h_{\mathrm{ra}}\left(V_{\mathrm{r}}[m]+U_{\mathrm{a}}[m]\right)+Z_{\mathrm{a}}[m] \\
Y_{\mathrm{b}}[m]=h_{\mathrm{rb}}\left(V_{\mathrm{r}}[m]+U_{\mathrm{a}}[m]\right)+Z_{\mathrm{b}}[m]
\end{array}\right.
$$

where $V_{\mathrm{r}}[m]$ and $U_{\mathrm{a}}[m]$ follow the distributions $V_{\mathrm{r}}^{(3)} \sim \mathcal{C N}\left(0, \beta P_{\mathrm{r}}\right), U_{\mathrm{a}}^{(3)} \sim \mathcal{C N}\left(0,(1-\beta) P_{\mathrm{r}}\right)$ respectively during phase $3, m \in\left[n\left(\Delta_{1, n}+\Delta_{2, n}\right)+1, n\right]$, where $(0 \leq \beta \leq 1)$ and $E\left[V_{\mathrm{r}}^{(3)} U_{\mathrm{b}}^{(3)}\right]=0$, i.e., $V_{\mathrm{r}}^{(3)}, U_{\mathrm{a}}^{(3)}$ are independent. We also take $\alpha=\frac{\left|h_{\mathrm{r}}\right|^{2} \beta P_{\mathrm{r}}}{\left|h_{\mathrm{r}}\right|^{2} \beta P_{\mathrm{r}}+1}$. Then

$$
\left\{\begin{array}{l}
I\left(U_{\mathrm{a}}^{(3)} ; Y_{\mathrm{b}}^{(3)}\right)=C\left(\frac{\left|h_{\mathrm{rb}}\right|^{2}(1-\beta) P_{\mathrm{r}}}{\left|h_{\mathrm{rb}}\right|^{2} \beta P_{\mathrm{r}}+1}\right) \\
I\left(U_{\mathrm{b}}^{(3)} ; Y_{\mathrm{a}}^{(3)}\right)-I\left(U_{\mathrm{a}}^{(3)} ; U_{\mathrm{b}}^{(3)}\right)=C\left(\left|h_{\mathrm{ra}}\right|^{2} \beta P_{\mathrm{r}}\right)
\end{array}\right.
$$

We similarly obtain the bounds for $\left|h_{\mathrm{ra}}\right| \leq\left|h_{\mathrm{rb}}\right|$. We note that the broadcast phase regions correspond to the capacity region of the Gaussian broadcast channel without own-message side-information (equations (15.11) and (15.12) in [4]). The following rates are achievable:

$$
\begin{aligned}
& R_{\mathrm{a}}<\Delta_{1} C\left(\left|h_{\mathrm{ab}}\right|^{2} P_{\mathrm{a}}+\frac{\left(\sigma_{y}^{(1)}\right)^{2}\left|h_{\mathrm{ar}}\right|^{2} P_{\mathrm{a}}}{P_{\hat{y}}^{(1)}\left(P_{y}^{(1)}\right)^{2}-\left(\sigma_{y}^{(1)}\right)^{2}\left(P_{y}^{(1)}-1\right)}\right) \\
& R_{\mathrm{b}}<\Delta_{2} C\left(\left|h_{\mathrm{ab}}\right|^{2} P_{\mathrm{b}}+\frac{\left(\sigma_{y}^{(2)}\right)^{2}\left|h_{\mathrm{br}}\right|^{2} P_{\mathrm{b}}}{P_{\hat{y}}^{(2)}\left(P_{y}^{(2)}\right)^{2}-\left(\sigma_{y}^{(2)}\right)^{2}\left(P_{y}^{(2)}-1\right)}\right)
\end{aligned}
$$

where,

$$
\text { if } \begin{aligned}
\left|h_{\mathrm{ra}}\right|<\left|h_{\mathrm{rb}}\right|: & \alpha_{\mathrm{a}} \Delta_{1} C\left(\frac{\left(\sigma_{y}^{(1)}\right)^{2}}{P_{\hat{y}}^{(1)} P_{y}^{(1)}-\left(\sigma_{y}^{(1)}\right)^{2}}\right)<\Delta_{3} C\left(\beta\left|h_{\mathrm{rb}}\right|^{2} P_{\mathrm{r}}\right) \\
& \alpha_{\mathrm{b}} \Delta_{2} C\left(\frac{\left(\sigma_{y}^{(2)}\right)^{2}}{P_{\hat{y}}^{(2)} P_{y}^{(2)}-\left(\sigma_{y}^{(2)}\right)^{2}}\right)<\Delta_{3} C\left(\frac{(1-\beta)\left|h_{\mathrm{ra}}\right|^{2} P_{\mathrm{r}}}{\beta\left|h_{\mathrm{ra}}\right|^{2} P_{\mathrm{r}}+1}\right), \\
\text { otherwise }: & \alpha_{\mathrm{a}} \Delta_{1} C\left(\frac{\left(\sigma_{y}^{(1)}\right)^{2}}{P_{\hat{y}}^{(1)} P_{y}^{(1)}-\left(\sigma_{y}^{(1)}\right)^{2}}\right)<\Delta_{3} C\left(\frac{(1-\beta)\left|h_{\mathrm{rb}}\right|^{2} P_{\mathrm{r}}}{\beta\left|h_{\mathrm{rb}}\right|^{2} P_{\mathrm{r}}+1}\right) \\
& \alpha_{\mathrm{b}} \Delta_{2} C\left(\frac{\left(\sigma_{y}^{(2)}\right)^{2}}{P_{\hat{y}}^{(2)} P_{y}^{(2)}-\left(\sigma_{y}^{(2)}\right)^{2}}\right)<\Delta_{3} C\left(\beta\left|h_{\mathrm{ra}}\right|^{2} P_{\mathrm{r}}\right),
\end{aligned}
$$


and

$$
\begin{aligned}
&\left(1-\alpha_{\mathrm{a}}\right) \Delta_{1} C\left(\frac{\left(\sigma_{y}^{(1)}\right)^{2}}{P_{\hat{y}}^{(1)} P_{y}^{(1)}-\left(\sigma_{y}^{(1)}\right)^{2}}\right)+\Delta_{2} C\left(\frac{\left(\sigma_{y}^{(2)}\right)^{2}}{P_{\hat{y}}^{(2)}\left(P_{y}^{(2)}\right)^{2}-\left(\sigma_{y}^{(2)}\right)^{2} P_{y}^{(2)}}\right) \\
&<\Delta_{4} C\left(\left|h_{\mathrm{br}}\right|^{2} P_{\mathrm{r}}\right)+\Delta_{1} C\left(\frac{\left(\sigma_{y}^{(1)}\right)^{2}\left|h_{\mathrm{ab}}\right|^{2}\left|h_{\mathrm{ra}}\right|^{2} P_{\mathrm{a}}}{\left(P_{y}^{(1)}\right)^{2} P_{\hat{y}}^{(1)}\left(\left|h_{\mathrm{ab}}\right|^{2} P_{\mathrm{a}}+1\right)-\left(\sigma_{y}^{(1)}\right)^{2}\left|h_{\mathrm{ab}}\right|^{2}\left|h_{\mathrm{ra}}\right|^{2} P_{\mathrm{a}}}\right) \\
&\left(1-\alpha_{\mathrm{b}}\right) \Delta_{2} C\left(\frac{\left(\sigma_{y}^{(2)}\right)^{2}}{P_{\hat{y}}^{(2)} P_{y}^{(2)}-\left(\sigma_{y}^{(2)}\right)^{2}}\right)+\Delta_{1} C\left(\frac{\left(\sigma_{y}^{(1)}\right)^{2}}{P_{\hat{y}}^{(1)}\left(P_{y}^{(1)}\right)^{2}-\left(\sigma_{y}^{(1)}\right)^{2} P_{y}^{(1)}}\right) \\
&<\Delta_{4} C\left(\left|h_{\mathrm{ar}}\right|^{2} P_{\mathrm{r}}\right)+\Delta_{2} C \\
& P_{y}^{(1)}=\left|h_{\mathrm{ar}}\right|^{2} P_{\mathrm{a}}+1 \\
& P_{y}^{(2)}=\left|h_{\mathrm{br}}\right|^{2} P_{\mathrm{b}}+1 \\
& 0<\alpha_{\mathrm{a}}, \alpha_{\mathrm{b}}, \beta<1 .
\end{aligned}
$$

Again, we numerically optimize $P_{\hat{y}}^{(\ell)}, \sigma_{y}^{(\ell)}, \Delta_{\ell}, \alpha_{\mathrm{a}}, \alpha_{\mathrm{b}}$ and $\beta$ to maximize the region's boundary.

\section{Lattice Forwarding}

The two-way relay channel is a canonical example for which, at least in full-duplex channels, the use of structured codes such as lattice codes for AWGN channels, is beneficial, particularly in highly symmetric high SNR scenarios. In [17], [18], [30] achievable rate regions for the full-duplex two-way relay channel using lattice codes are derived. The structure offered by lattice codes allows for a sum of the messages to be decoded at the relay, forming a type of lattice-based Decode and Forward scheme. Prior work has considered full-duplex rate regions employing lattice codes without [17], [18], and recently with [30] direct links. We adapt the scheme of [17] to the case in which nodes are half-duplex and follow an MABC protocol. We note that for the full duplex scheme with direct links of [30], the direct link cannot be used in an MABC-like fashion due to the half-duplex nature of the nodes and as such the benefits over the scheme of [17] under MABC constraints disappears.

\section{- MABC Protocol}

The focus of this work is not on lattice codes; we do however state a new region for the half-duplex DF MABC protocol which employs lattices at the terminal nodes. This region is derived directly from that of [17] by taking the two phases into account; its proof follows immediately from [17] and is omitted. One may show that the following rates may be achieved using lattice codes: 


$$
\begin{aligned}
& R_{\mathrm{a}}<\min \left\{\left[\Delta_{1} \log \left(\frac{P_{\mathrm{a}}}{P_{\mathrm{a}}+P_{\mathrm{b}}}+\left|h_{\mathrm{ar}}\right|^{2} P_{\mathrm{a}}\right)\right]^{+}, \Delta_{2} C\left(\left|h_{\mathrm{rb}}\right|^{2} P_{\mathrm{r}}\right)\right\} \\
& R_{\mathrm{b}}<\min \left\{\left[\Delta_{1} \log \left(\frac{P_{\mathrm{b}}}{P_{\mathrm{a}}+P_{\mathrm{b}}}+\left|h_{\mathrm{br}}\right|^{2} P_{\mathrm{b}}\right)\right]^{+}, \Delta_{2} C\left(\left|h_{\mathrm{ra}}\right|^{2} P_{\mathrm{r}}\right)\right\} .
\end{aligned}
$$

\section{- TDBC protocol}

One may derive an achievable rate region for the DF TDBC protocol in which terminal nodes employ lattice codes. However, this would not improve the rate region over a random-coding-based region as the gains of lattice codes stem from removing the multiple-access-like constraints at the relay node (i.e. removing the sum-rate constraint); in a TDBC protocol this multiple access phase does not exist and as such we do not present an achievable rate region for the Lattice-based TDBC protocol.

\section{E. Mixed Forward}

Applying Theorem 3 to the Gaussian case with Costa's setup in [3] the channel in the relay broadcast phase for the Mixed MABC protocol is given by:

$$
\text { In phase } 2\left\{\begin{array}{l}
U_{\mathrm{r}}[m]=V_{\mathrm{r}}[m]+\alpha U_{\mathrm{b}}[m] \\
Y_{\mathrm{a}}[m]=h_{\mathrm{ra}}\left(V_{\mathrm{r}}[m]+U_{\mathrm{b}}[m]\right)+Z_{\mathrm{a}}[m] \\
Y_{\mathrm{b}}[m]=h_{\mathrm{rb}}\left(V_{\mathrm{r}}[m]+U_{\mathrm{b}}[m]\right)+Z_{\mathrm{b}}[m]
\end{array}\right.
$$

where $V_{\mathrm{r}}[m]$ and $U_{\mathrm{b}}[m]$ follow $V_{\mathrm{r}}^{(2)} \sim \mathcal{C N}\left(0, \beta P_{\mathrm{r}}\right), U_{\mathrm{b}}^{(2)} \sim \mathcal{C N}\left(0,(1-\beta) P_{\mathrm{r}}\right)$ during phase $2, m \in$ $\left[\Delta_{1, n} \cdot n+1, n\right], 0 \leq \beta \leq 1$, and $V_{\mathrm{r}}^{(2)}, U_{\mathrm{b}}^{(2)}$ are independent. Then we obtain the following achievable rate region, where we numerically optimize $\alpha, \beta, P_{\hat{y}}^{(\ell)}, \sigma_{y}^{(\ell)}$ and $\Delta_{\ell}$ to maximize the boundary.

- TDBC Protocol

$$
\begin{aligned}
& R_{\mathrm{a}}<\min \left\{\Delta_{1} C\left(\left|h_{\mathrm{ar}}\right|^{2} P_{\mathrm{a}}\right),\right. \\
& \left.\Delta_{1} C\left(\left|h_{\mathrm{ab}}\right|^{2} P_{\mathrm{a}}\right)+\Delta_{3} \log _{2}\left(\frac{\beta P_{\mathrm{r}}\left(\left|h_{\mathrm{rb}}\right|^{2} P_{\mathrm{r}}+1\right)}{\left|h_{\mathrm{rb}}\right|^{2}(1-\alpha)^{2} \beta(1-\beta) P_{\mathrm{r}}^{2}+\beta P_{\mathrm{r}}+\alpha^{2}(1-\beta) P_{\mathrm{r}}}\right)\right\} \\
& R_{\mathrm{b}}<\Delta_{2} C\left(\left|h_{\mathrm{ab}}\right|^{2} P_{\mathrm{b}}+\frac{\left(\sigma_{y}^{(2)}\right)^{2}\left|h_{\mathrm{br}}\right|^{2} P_{\mathrm{b}}}{P_{\hat{y}}^{(2)}\left(P_{y}^{(2)}\right)^{2}-\left(\sigma_{y}^{(2)}\right)^{2} P_{y}^{(2)}+\left(\sigma_{y}^{(2)}\right)^{2}}\right)
\end{aligned}
$$


where,

$$
\begin{aligned}
\Delta_{2} C\left(\frac{\left(\sigma_{y}^{(2)}\right)^{2}\left(1-P^{*}\right)}{P_{\hat{y}}^{(2)} P_{y}^{(2)}-\left(\sigma_{y}^{(2)}\right)^{2}}\right) & <\min \left\{\Delta_{3} C\left(\left|h_{\mathrm{ra}}\right|^{2} P_{\mathrm{r}}\right), \Delta_{3} C\left(\left|h_{\mathrm{ra}}\right|^{2}(1-\alpha)^{2}(1-\beta) P_{\mathrm{r}}+\frac{\alpha^{2}(1-\beta)}{\beta}\right)\right\} \\
P_{y}^{(2)} & =\left|h_{\mathrm{br}}\right|^{2} P_{\mathrm{b}}+1 \\
P^{*} & =\frac{\left|h_{\mathrm{ab}}\right|^{2} P_{\mathrm{b}}}{\left|h_{\mathrm{ab}}\right|^{2} P_{\mathrm{b}}+1} \cdot \frac{\left|h_{\mathrm{rb}}\right|^{2} P_{\mathrm{b}}}{\left|h_{\mathrm{rb}}\right|^{2} P_{\mathrm{b}}+1}
\end{aligned}
$$

- MABC Protocol

The Mixed MABC protocol is not presented separately as it was shown be contained in the DF MABC region.

\section{F. Outer Bound}

Applying Theorems 2 and 4 in [13] to the Gaussian case, we obtain the following outer bounds. We optimize $\Delta_{\ell}$ 's for given channel gains (and hence given mutual information expressions) to maximize these outer bounds.

- MABC Protocol

$$
\begin{aligned}
& R_{\mathrm{a}} \leq \min \left\{\Delta_{1} C\left(\left|h_{\mathrm{ar}}\right|^{2} P_{\mathrm{a}}\right), \Delta_{2} C\left(\left|h_{\mathrm{br}}\right|^{2} P_{\mathrm{r}}\right)\right\} \\
& R_{\mathrm{b}} \leq \min \left\{\Delta_{1} C\left(\left|h_{\mathrm{br}}\right|^{2} P_{\mathrm{b}}\right), \Delta_{2} C\left(\left|h_{\mathrm{ar}}\right|^{2} P_{\mathrm{r}}\right)\right\}
\end{aligned}
$$

- TDBC Protocol

$$
\begin{aligned}
R_{\mathrm{a}} & \leq \min \left\{\Delta_{1} C\left(\left|h_{\mathrm{ar}}\right|^{2} P_{\mathrm{a}}+\left|h_{\mathrm{ab}}\right|^{2} P_{\mathrm{a}}\right), \Delta_{1} C\left(\left|h_{\mathrm{ab}}\right|^{2} P_{\mathrm{a}}\right)+\Delta_{3} C\left(\left|h_{\mathrm{br}}\right|^{2} P_{\mathrm{r}}\right)\right\} \\
R_{\mathrm{b}} & \leq \min \left\{\Delta_{2} C\left(\left|h_{\mathrm{br}}\right|^{2} P_{\mathrm{b}}+\left|h_{\mathrm{ab}}\right|^{2} P_{\mathrm{b}}\right), \Delta_{2} C\left(\left|h_{\mathrm{ab}}\right|^{2} P_{\mathrm{b}}\right)+\Delta_{3} C\left(\left|h_{\mathrm{ar}}\right|^{2} P_{\mathrm{r}}\right)\right\} \\
R_{\mathrm{a}}+R_{\mathrm{b}} & \leq \Delta_{1} C\left(\left|h_{\mathrm{ar}}\right|^{2} P_{\mathrm{a}}\right)+\Delta_{2} C\left(\left|h_{\mathrm{br}}\right|^{2} P_{\mathrm{b}}\right)
\end{aligned}
$$

\section{AChiEvable Rate Regions in the GaUssian CHANNEL}

In order to obtain an intuitive feel for the regions and to illustrate that the regions are not subsets of one another, the bounds described in Section IV are plotted in this section for a number of different channel configurations. We first compare the rate regions obtained by the bi-directional protocols and outer bounds in cases in which the links are symmetric $\left(h_{\mathrm{ar}}=h_{\mathrm{br}}=1, h_{\mathrm{ab}}=0.2\right)$ as well as asymmetric $\left(h_{\mathrm{ar}}=0.6, h_{\mathrm{br}}=20, h_{\mathrm{ab}}=0.5\right.$ and $\left.h_{\mathrm{ar}}=20, h_{\mathrm{br}}=0.6, h_{\mathrm{ab}}=0.5\right)$ for transmit SNRs of 0 and $20 \mathrm{~dB}$. We then proceed to examine the maximal sum-rate $R_{\mathrm{a}}+R_{\mathrm{b}}$ of the 10 schemes ( 8 achievable rate regions 
and 2 outer bounds) as a function of the transmit SNR. We find that different schemes are optimal under different channel conditions. We provide further discussions in the following subsections.

\section{A. Achievable rate region comparisons}

1) Symmetric Case: In this case $h_{\mathrm{ar}}=h_{\mathrm{br}}=1$ (Figs. 2, 3). In the low SNR regime, the DF MABC protocol dominates the other protocols. The MABC protocol in general outperforms the TDBC protocol as the benefits of side information and reduced interference are relatively small in this regime. The DF scheme outperforms the other schemes since the relatively large amount of noise in the first phase (and the second phase in the TDBC protocol) can be eliminated in the DF scheme, which cannot be done using the other schemes. In contrast, the DF TDBC protocol dominates the other protocols at high SNR since the direct link is strong enough to convey information in this regime. In the high SNR regime (when $P_{\mathrm{a}}=P_{\mathrm{b}}=P_{\mathrm{r}}=P$ is sufficiently large), the Lattice MABC protocol outperforms the CF, AF and random-coding based DF MABC protocols. Furthermore, from [17] it may be shown that the achievable region of the Lattice MABC protocol is within 1 bit of the MABC outer bound regardless of channel conditions. In the TDBC protocol, the CF scheme does not outperform the DF scheme as the DF uses two parallel channels in phase one and three while CF uses one channel in phase one with two receivers. In other words, $R_{\mathrm{a}}^{D F}<\Delta_{1} C(\cdot)+\Delta_{3} C(\cdot)$ for the DF as opposed to $R_{\mathrm{a}}^{C F}<\Delta_{1} C\left(\sum \cdot\right)$ for the CF scheme. However, under the MABC protocol, the CF scheme outperforms DF in the high SNR regime. This is because the interference of the transmission of two terminal nodes affects the DF MABC scheme due to the multiple-access nature but not the CF scheme (as it does not decode the signals). In Figs. 2 and 3, AF is always outer bounded by the CF scheme. We thus expect that when relay $r$ does not know the full codebooks of $a$ and $b$ (and hence cannot decode as in the DF scheme), that CF (in which some compression codebook knowledge is assumed at the relay) is a better choice than the AF scheme. In the low SNR regime, the achievable rate region of the DF MABC protocol and the outer bound of the MABC protocol are visibly tight, while in the high SNR regime, the achievable rate region of the Lattice $\mathrm{DF}$ and $\mathrm{CF}$ MABC protocols are tight. For the TDBC protocol, there is a very small gap between the achievable rate region of the DF TDBC protocol and the TDBC outer bound since interference is not present in the TDBC protocol. Hence decoding at the relay is intuitively, at least, nearly optimal.

2) Asymmetric Cases: In these cases $h_{\mathrm{ar}}=0.6, h_{\mathrm{br}}=20, h_{\mathrm{ab}}=0.5$ (Figs. 4,5$)$ and $h_{\mathrm{ar}}=20, h_{\mathrm{br}}=$ $0.6, h_{\mathrm{ab}}=0.5$ (Figs. 6, 7). Note that these two asymmetric cases are different for the mixed forwarding scheme, which assumes CF in one direction and DF in the other. In the low SNR regime, the CF TDBC 


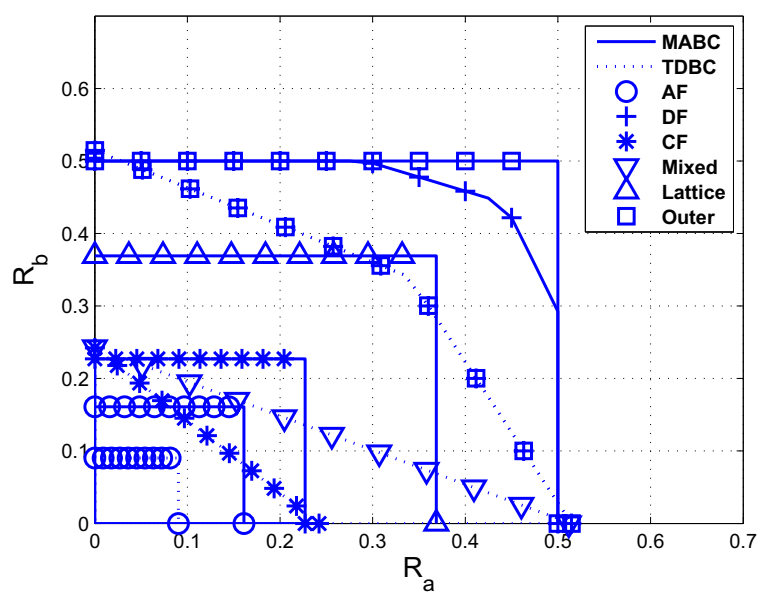

Fig. 2. Comparison of bi-directional regions with $h_{\mathrm{ar}}=$ $h_{\mathrm{br}}=1, h_{\mathrm{ab}}=0.2, P_{\mathrm{a}}=P_{\mathrm{b}}=P_{\mathrm{r}}=0 \mathrm{~dB}$ and $N_{\mathrm{a}}=N_{\mathrm{b}}=$ $N_{\mathrm{r}}=1$.

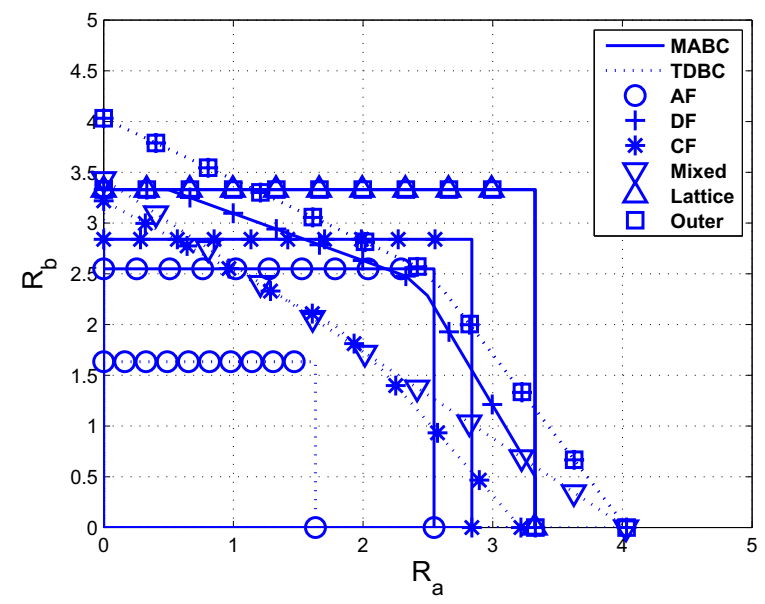

Fig. 3. Comparison of bi-directional regions with $h_{\mathrm{ar}}=$ $h_{\mathrm{br}}=1, h_{\mathrm{ab}}=0.2, P_{\mathrm{a}}=P_{\mathrm{b}}=P_{\mathrm{r}}=20 \mathrm{~dB}$ and $N_{\mathrm{a}}=$ $N_{\mathrm{b}}=N_{\mathrm{r}}=1$.

and mixed TDBC protocols achieve the best performance in Fig. 4 and Fig. 6, respectively. However, in the high SNR regime, the DF and Lattice MABC protocols and the DF TDBC protocol yields larger regions than the other protocols. In contrast to the symmetric case, the AF MABC protocol is not outer bounded by the CF MABC protocol. We note that the mixed forwarding scheme is the only one in which the relative performance of the schemes changes depending on which of the asymmetric scenarios is considered. In particular, in the mixed TDBC protocol, if $h_{\mathrm{ar}}>h_{\mathrm{br}}$, we obtain a larger achievable rate region than the plain DF TDBC protocol as the first link is more critical to the performance of the DF scheme. As the SNR increases, the difference between the two asymmetric cases decreases.

\section{B. Maximum Sum Data Rate}

In this subsection we plot the maximum sum-rate $R_{\mathrm{a}}+R_{\mathrm{b}}$ as a function of the transmit SNR for the symmetric case of the previous subsection. As expected, different schemes dominate for different SNR values. The sum-rate is proportional to the SNR in $\mathrm{dB}$ scale since the sum-rate is roughly the logarithm of the SNR. At high SNR, the Lattice-based MABC protocol very closely approximates the outer bound. As discussed in the previous subsection, the achievable rate region of the Lattice MABC protocol is within 1 bit of the MABC outer bound. In Fig. 8 at around $12 \mathrm{~dB}$ the relative performance of the $\mathrm{CF}$ MABC protocol and the DF MABC protocol changes. At lower SNRs, the DF MABC protocol is better, while at higher SNRs, the Lattice and CF MABC protocols are better. We also note that the AF MABC protocol is always worse than the CF MABC protocol in the symmetric case (Fig. 8). In the TDBC 


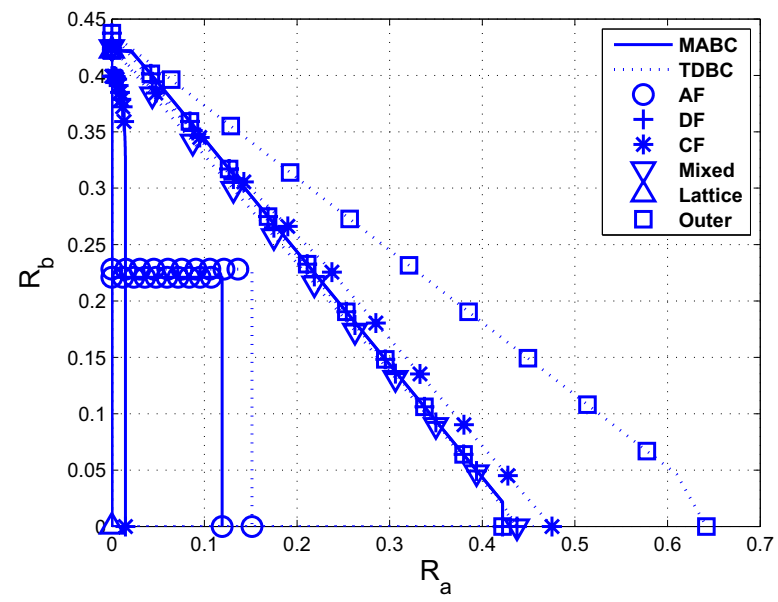

Fig. 4. Comparison of bi-directional regions with $h_{\mathrm{ar}}=$ $0.6, h_{\mathrm{br}}=20, h_{\mathrm{ab}}=0.5, P_{\mathrm{a}}=P_{\mathrm{b}}=P_{\mathrm{r}}=0 \mathrm{~dB}$ and $N_{\mathrm{a}}=N_{\mathrm{b}}=N_{\mathrm{r}}=1$.

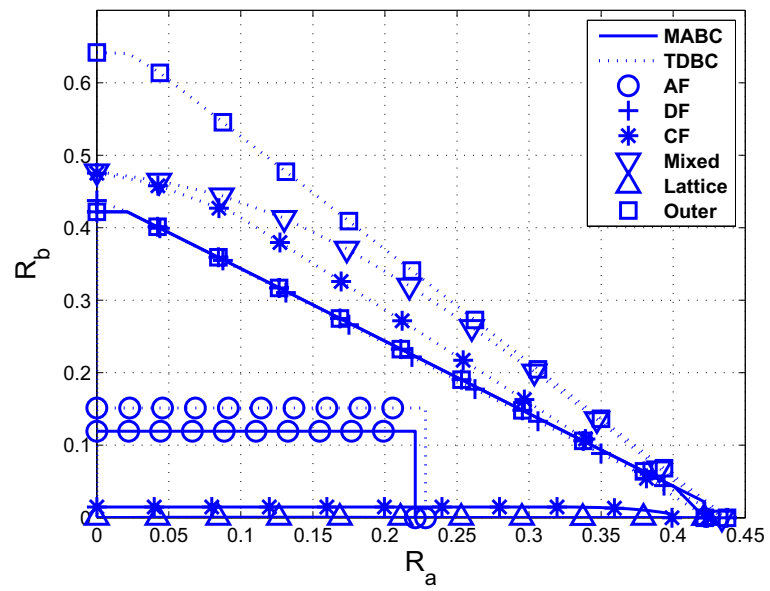

Fig. 6. Comparison of bi-directional regions with $h_{\text {ar }}=20$, $h_{\mathrm{br}}=0.6, h_{\mathrm{ab}}=0.5, P_{\mathrm{a}}=P_{\mathrm{b}}=P_{\mathrm{r}}=0 \mathrm{~dB}$ and $N_{\mathrm{a}}=$ $N_{\mathrm{b}}=N_{\mathrm{r}}=1$.

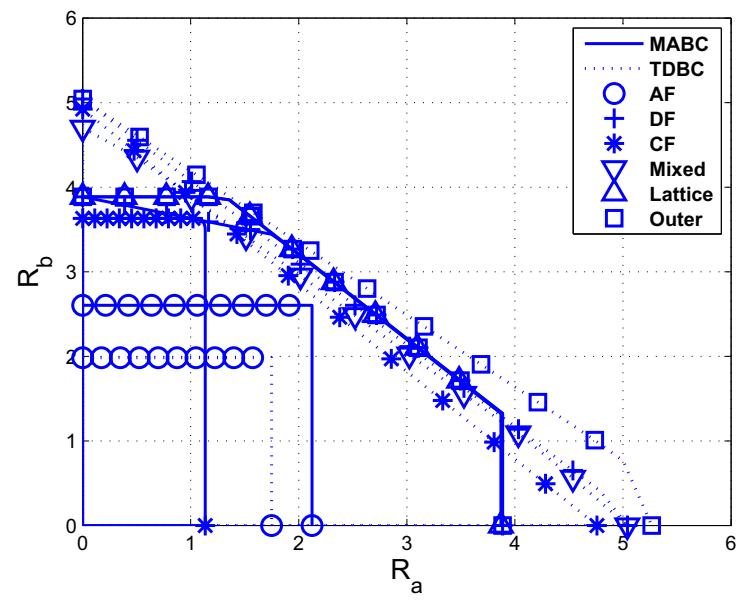

Fig. 5. Comparison of bi-directional regions with $h_{\mathrm{ar}}=$ $0.6, h_{\mathrm{br}}=20, h_{\mathrm{ab}}=0.5, P_{\mathrm{a}}=P_{\mathrm{b}}=P_{\mathrm{r}}=20 \mathrm{~dB}$ and $N_{\mathrm{a}}=N_{\mathrm{b}}=N_{\mathrm{r}}=1$.

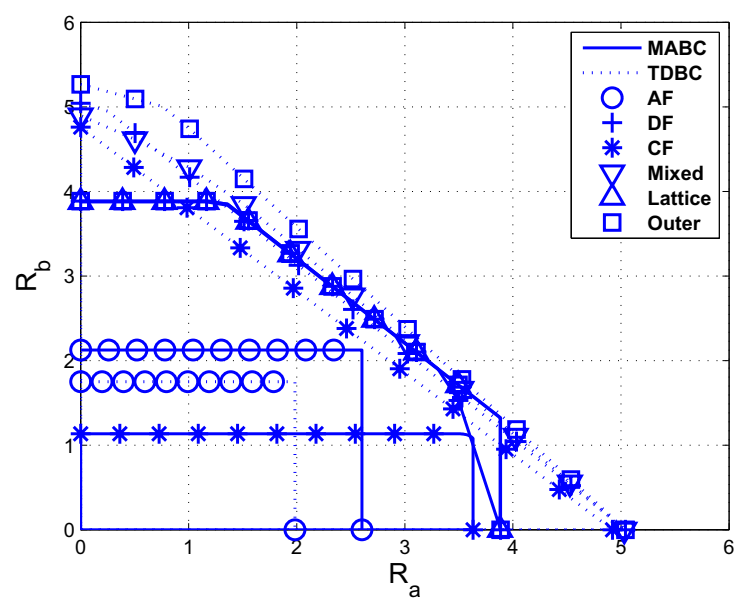

Fig. 7. Comparison of bi-directional regions with $h_{\mathrm{ar}}=20$, $h_{\mathrm{br}}=0.6, h_{\mathrm{ab}}=0.5, P_{\mathrm{a}}=P_{\mathrm{b}}=P_{\mathrm{r}}=20 \mathrm{~dB}$ and $N_{\mathrm{a}}=$ $N_{\mathrm{b}}=N_{\mathrm{r}}=1$.

protocol, the sum-rate of the mixed TDBC protocol lies between the DF scheme and the CF scheme in Fig. 8.

\section{CONCLUSION}

In this paper, we have derived achievable rate regions for 4 new half-duplex bi-directional relaying protocols and have provided a comprehensive numerical comparison of the half-duplex two-way achiev- 


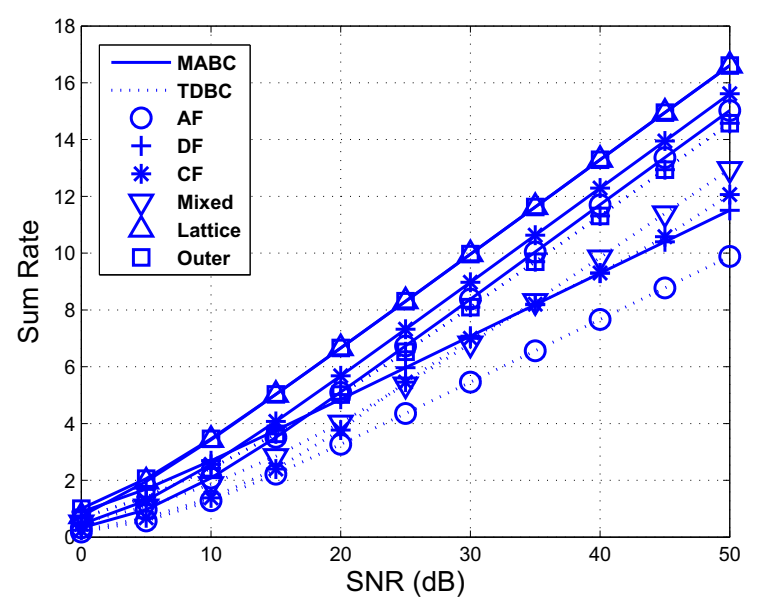

Fig. 8. Maximum sum-rate of the 8 bi-directional protocols and 2 outer bounds at different SNR. Here $h_{\mathrm{ar}}=h_{\mathrm{br}}=1$ and $h_{\mathrm{ab}}=0.2$

able rate regions and outer bounds assuming Gaussian input distributions in AWGN channels. For the MABC protocol, DF (with random or lattice codebooks) or CF is the optimal scheme, depending on the given channel and SNR regime. In particular, the new half-duplex lattice DF MABC protocol performs well for symmetric, high SNR channels. In asymmetric cases, Mixed protocols which employ DF in one direction and $\mathrm{CF}$ in the other perform well, as different forwarding schemes in the two directions may be more tailored to the different channel conditions in the two directions. In the TDBC protocol, the relative performance of the forwarding schemes depends on the given channel conditions. Notably, we have determined an example of a channel condition in which the mixed TDBC protocol outperforms the other proposed protocols. In general, the MABC protocol outperforms the TDBC protocol in the low SNR regime, while the reverse is true in the high SNR regime.

\section{APPENDIX A}

\section{PROOF OF THEOREM 1}

Proof: Random code generation: For simplicity of exposition, we take $|\mathcal{Q}|=1$. For any sequence $\Delta_{\ell, n}$ converging to $\Delta_{\ell}$ :

1) Phase 1: Generate random $\left(n \cdot \Delta_{1, n}\right)$-length sequences

- $\mathbf{x}_{\mathrm{a}}^{(1)}\left(w_{\mathrm{a}}\right)$ i.i.d. with $p^{(1)}\left(x_{\mathrm{a}}\right), w_{\mathrm{a}} \in \mathcal{S}_{\mathrm{a}}=\left\{0,1, \cdots,\left\lfloor 2^{n R_{\mathrm{a}}}\right\rfloor-1\right\}$

- $\hat{\mathbf{y}}_{\mathrm{r}}^{(1)}\left(w_{\mathrm{a} 0}\right)$ i.i.d. with $p^{(1)}\left(\hat{y}_{\mathrm{r}}\right)=\sum_{y_{\mathrm{r}}} p^{(1)}\left(y_{\mathrm{r}}\right) p^{(1)}\left(\hat{y}_{\mathrm{r}} \mid y_{\mathrm{r}}\right), w_{\mathrm{a} 0} \in\left\{0,1, \cdots,\left\lfloor 2^{n R_{\mathrm{a} 0}}\right\rfloor-1\right\}=: \mathcal{S}_{\mathrm{a} 0}$ 
and generate a partition of $\mathcal{S}_{\mathrm{a} 0}$ randomly by independently assigning every index $w_{\mathrm{a} 0} \in \mathcal{S}_{\mathrm{a} 0}$ to a set $\mathcal{S}_{\mathrm{a} 0, i}$, with a uniform distribution over the indices $i \in\left\{0, \ldots,\left\lfloor 2^{n R_{\mathrm{a} 1}}\right\rfloor-1\right\}:=\mathcal{S}_{\mathrm{a} 1}$. We denote by $s_{\mathrm{a} 0}\left(w_{\mathrm{a} 0}\right)$ the index $i$ of $\mathcal{S}_{\mathrm{a} 0, i}$ to which $w_{\mathrm{a} 0}$ belongs.

2) Phase 2: Generate random $\left(n \cdot \Delta_{2, n}\right)$-length sequences

- $\mathbf{x}_{\mathrm{b}}^{(2)}\left(w_{\mathrm{b}}\right)$ i.i.d. with $p^{(2)}\left(x_{\mathrm{b}}\right), w_{\mathrm{b}} \in \mathcal{S}_{\mathrm{b}}=\left\{0,1, \cdots,\left\lfloor 2^{n R_{\mathrm{b}}}\right\rfloor-1\right\}$

- $\hat{\mathbf{y}}_{\mathrm{r}}^{(2)}\left(w_{\mathrm{b} 0}\right)$ i.i.d. with $p^{(2)}\left(\hat{y}_{\mathrm{r}}\right)=\sum_{y_{\mathrm{r}}} p^{(2)}\left(y_{\mathrm{r}}\right) p^{(2)}\left(\hat{y}_{\mathrm{r}} \mid y_{\mathrm{r}}\right), w_{\mathrm{b} 0} \in\left\{0,1, \cdots,\left\lfloor 2^{n R_{\mathrm{b} 0}}\right\rfloor-1\right\}=: \mathcal{S}_{\mathrm{b} 0}$ and generate a partition of $\mathcal{S}_{\mathrm{b} 0}$ randomly by independently assigning every index $w_{\mathrm{b} 0} \in \mathcal{S}_{\mathrm{b} 0}$ to a set $\mathcal{S}_{\mathrm{b} 0, i}$, with a uniform distribution over the indices $i \in\left\{0, \ldots,\left\lfloor 2^{n R_{\mathrm{b} 1}}\right\rfloor-1\right\}=: \mathcal{S}_{\mathrm{b} 1}$. We denote by $s_{\mathrm{b} 0}\left(w_{\mathrm{b} 0}\right)$ the index $i$ of $\mathcal{S}_{\mathrm{b} 0, i}$ to which $w_{\mathrm{b} 0}$ belongs.

3) Phase 3: Generate random $\left(n \cdot \Delta_{3, n}\right)$-length sequences

- $\mathbf{u}_{\mathrm{a}}^{(3)}\left(w_{\mathrm{a} 2}\right)$ i.i.d with $p^{(3)}\left(u_{\mathrm{a}}\right), w_{\mathrm{a} 2} \in\left\{0,1, \cdots,\left\lfloor 2^{n R_{\mathrm{a} 2}}\right\rfloor-1\right\}=: \mathcal{S}_{\mathrm{a} 2}$

- $\mathbf{u}_{\mathrm{b}}^{(3)}\left(w_{\mathrm{b} 2}\right)$ i.i.d with $p^{(3)}\left(u_{\mathrm{b}}\right), w_{\mathrm{b} 2} \in\left\{0,1, \cdots,\left\lfloor 2^{n R_{\mathrm{b} 2}}\right\rfloor-1\right\}=: \mathcal{S}_{\mathrm{b} 2}$

and define bin $B_{j}:=\left\{w_{\mathrm{a} 2} \mid w_{\mathrm{a} 2} \in\left[(j-1) \cdot\left\lfloor 2^{n\left(R_{\mathrm{a} 2}-R_{\mathrm{a} 1}\right)}\right\rfloor+1, j \cdot\left\lfloor 2^{n\left(R_{\mathrm{a} 2}-R_{\mathrm{a} 1}\right)}\right\rfloor\right]\right\}$ for $j \in \mathcal{S}_{\mathrm{a} 1}$. Likewise, $C_{k}:=\left\{w_{\mathrm{b} 2} \mid w_{\mathrm{b} 2} \in\left[(k-1) \cdot\left\lfloor 2^{n\left(R_{\mathrm{b} 2}-R_{\mathrm{b} 1}\right)}\right\rfloor+1, k \cdot\left\lfloor 2^{n\left(R_{\mathrm{b} 2}-R_{\mathrm{b} 1}\right)}\right\rfloor\right]\right\}$ for $k \in \mathcal{S}_{\mathrm{b} 1}$.

4) Phase 4: Generate random $\left(n \cdot \Delta_{4, n}\right)$-length sequences

- $\mathbf{x}_{\mathrm{r}}^{(4)}\left(w_{\mathrm{a} 0}, w_{\mathrm{b} 0}\right)$ i.i.d with $p^{(4)}\left(x_{\mathrm{r}}\right), w_{\mathrm{a} 0} \in \mathcal{S}_{\mathrm{a} 0}$ and $w_{\mathrm{b} 0} \in \mathcal{S}_{\mathrm{b} 0}$.

Encoding: During phase 1 (resp. phase 2), the encoder of node a (resp. b) sends the codeword $\mathbf{x}_{\mathbf{a}}^{(1)}\left(w_{\mathrm{a}}\right)$ (resp. $\mathbf{x}_{\mathbf{b}}^{(2)}\left(w_{\mathbf{b}}\right)$ ). At the end of phase 1 , relay $r$ compresses the received signal $\mathbf{y}_{\mathbf{r}}^{(1)}$ into the message $w_{\mathrm{a} 0}$ if there exists a $w_{\mathrm{a} 0}$ such that $\left(\mathbf{y}_{\mathrm{r}}^{(1)}, \hat{\mathbf{y}}_{\mathrm{r}}^{(1)}\left(w_{\mathrm{a} 0}\right)\right) \in A^{(1)}\left(Y_{\mathrm{r}} \hat{Y}_{\mathrm{r}}\right)$. Similarly, $r$ compresses $\mathbf{y}_{\mathrm{r}}^{(2)}$ into the message $w_{\mathrm{b} 0}$ at the end of phase 2 . There exist such $w_{\mathrm{a} 0}$ and $w_{\mathrm{b} 0}$ with high probability if

$$
\begin{aligned}
& R_{\mathrm{a} 0}=\Delta_{1, n} I\left(Y_{\mathrm{r}}^{(1)} ; \hat{Y}_{\mathrm{r}}^{(1)}\right)+\epsilon \\
& R_{\mathrm{b} 0}=\Delta_{2, n} I\left(Y_{\mathrm{r}}^{(2)} ; \hat{Y}_{\mathrm{r}}^{(2)}\right)+\epsilon
\end{aligned}
$$

and $n$ is sufficiently large. We choose $R_{\mathrm{a} 1}, R_{\mathrm{b} 1}, R_{\mathrm{a} 2}$ and $R_{\mathrm{b} 2}$ as:

$$
\begin{aligned}
& R_{\mathrm{a} 1}=\alpha_{\mathrm{a}} R_{\mathrm{a} 0}=\alpha_{\mathrm{a}}\left(\Delta_{1, n} I\left(Y_{\mathrm{r}}^{(1)} ; \hat{Y}_{\mathrm{r}}^{(1)}\right)+\epsilon\right) \\
& R_{\mathrm{b} 1}=\alpha_{\mathrm{b}} R_{\mathrm{b} 0}=\alpha_{\mathrm{b}}\left(\Delta_{2, n} I\left(Y_{\mathrm{r}}^{(2)} ; \hat{Y}_{\mathrm{r}}^{(2)}\right)+\epsilon\right)
\end{aligned}
$$

and

$$
\begin{aligned}
& R_{\mathrm{a} 1} \leq R_{\mathrm{a} 2}=\Delta_{3, n} I\left(U_{\mathrm{a}}^{(3)} ; Y_{\mathrm{b}}^{(3)}\right)-4 \epsilon \\
& R_{\mathrm{b} 1} \leq R_{\mathrm{b} 2}=\Delta_{3, n} I\left(U_{\mathrm{b}}^{(3)} ; Y_{\mathrm{a}}^{(3)}\right)-4 \epsilon .
\end{aligned}
$$


From the code constructions of $w_{\mathrm{a} 2}$ and $w_{\mathrm{b} 2}, R_{\mathrm{a} 1}$ and $R_{\mathrm{b} 1}$ have to be less than $R_{\mathrm{a} 2}$ and $R_{\mathrm{b} 2}$, respectively. Then the relay constructs $w_{\mathrm{a} 1}=s_{\mathrm{a} 0}\left(w_{\mathrm{a} 0}\right)$ and $w_{\mathrm{b} 1}=s_{\mathrm{b} 0}\left(w_{\mathrm{b} 0}\right)$. To choose $w_{\mathrm{a} 2}$ and $w_{\mathrm{b} 2}$, the relay first selects the bins $B_{w_{\mathrm{a} 1}}$ and $C_{w_{\mathrm{b} 1}}$ and then it searches for a pair $\left(w_{\mathrm{a} 2}, w_{\mathrm{b} 2}\right) \in B_{w_{\mathrm{a} 1}} \times C_{w_{\mathrm{b} 1}}$ such that $\left(\mathbf{u}_{\mathrm{a}}^{(3)}\left(w_{\mathrm{a} 2}\right), \mathbf{u}_{\mathrm{b}}^{(3)}\left(w_{\mathrm{b} 2}\right)\right) \in A^{(3)}\left(U_{\mathrm{a}} U_{\mathrm{b}}\right)$. Such a $\left(w_{\mathrm{a} 2}, w_{\mathrm{b} 2}\right)$ exists with high probability if

$$
R_{\mathrm{a} 1}+R_{\mathrm{b} 1}<R_{\mathrm{a} 2}+R_{\mathrm{b} 2}-\Delta_{3, n} I\left(U_{\mathrm{a}}^{(3)} ; U_{\mathrm{b}}^{(3)}\right)-\epsilon^{\prime}
$$

from the Lemma in [8]. The relay then sends $\mathbf{x}_{\mathbf{r}}^{(3)}$ generated i.i.d. according to $p^{(3)}\left(x_{\mathbf{r}} \mid u_{\mathrm{a}}, u_{\mathbf{b}}\right)$ with $\mathbf{u}_{\mathrm{a}}^{(3)}\left(w_{\mathrm{a} 2}\right)$ and $\mathbf{u}_{\mathrm{b}}^{(3)}\left(w_{\mathrm{b} 2}\right)$ during phase 3. Finally, the relay sends $\mathbf{x}_{\mathrm{r}}^{(4)}\left(w_{\mathrm{a} 0}, w_{\mathrm{b} 0}\right)$ during phase 4 .

Decoding: Node a decodes $\tilde{w}_{\mathrm{b} 2}$ after phase 3 using jointly typical decoding. Then a estimates $\tilde{w}_{\mathrm{b} 1}$ from the bin index of $\tilde{w}_{\mathrm{b} 2}$. Node a decodes $\tilde{w}_{\mathrm{b} 0}$ if there exists a unique $\tilde{w}_{\mathrm{b} 0}$ such that $\tilde{w}_{\mathrm{b} 0} \in \mathcal{S}_{\mathrm{b} 0, \tilde{w}_{\mathrm{b} 1}}$, $\left(\mathbf{x}_{\mathrm{r}}^{(4)}\left(\tilde{w}_{\mathrm{a} 0}, \tilde{w}_{\mathrm{b} 0}\right), \mathbf{y}_{\mathrm{a}}^{(4)}\right) \in A^{(4)}\left(X_{\mathrm{r}} Y_{\mathrm{a}}\right),\left(\mathbf{x}_{\mathrm{a}}^{(1)}\left(w_{\mathrm{a}}\right), \hat{\mathbf{y}}_{\mathrm{r}}^{(1)}\left(\tilde{w}_{\mathrm{a} 0}\right)\right) \in A^{(1)}\left(X_{\mathrm{a}} \hat{Y}_{\mathrm{r}}\right)$ and $\left(\hat{\mathbf{y}}_{\mathrm{b}}^{(2)}\left(w_{\mathrm{b} 0}\right), \mathbf{y}_{\mathrm{a}}^{(2)}\right) \in A^{(2)}\left(\hat{Y}_{\mathrm{r}} Y_{\mathrm{a}}\right)$ . After decoding $\tilde{w}_{\mathrm{b} 0}$, node a decodes $\tilde{w}_{\mathrm{b}}$ using jointly typical decoding of the sequence $\left(\mathbf{x}_{\mathrm{b}}^{(2)}, \hat{\mathbf{y}}_{\mathbf{r}}^{(2)}\left(\tilde{w}_{\mathrm{b} 0}\right), \mathbf{y}_{\mathrm{a}}^{(2)}\right)$. Similarly, node b decodes $\tilde{w}_{\mathrm{a}}$. Error analysis:

$$
\begin{aligned}
P\left[E_{\mathrm{b}, \mathrm{a}}\right] & \leq P\left[E_{\mathrm{r}, \mathrm{a}}^{(3)} \cup E_{\mathrm{r}, \mathrm{a}}^{(4)} \cup E_{\mathrm{b}, \mathrm{a}}^{(4)}\right] \\
& \leq P\left[E_{\mathrm{r}, \mathrm{a}}^{(3)}\right]+P\left[E_{\mathrm{r}, \mathrm{a}}^{(4)} \mid \bar{E}_{\mathrm{r}, \mathrm{a}}^{(3)}\right]+P\left[E_{\mathrm{b}, \mathrm{a}}^{(4)} \mid \bar{E}_{\mathrm{r}, \mathrm{a}}^{(3)} \cap \bar{E}_{\mathrm{r}, \mathrm{a}}^{(4)}\right]
\end{aligned}
$$

Then,

$$
\begin{aligned}
P\left[E_{\mathrm{r}, \mathrm{a}}^{(3)}\right] \leq & P\left[\cup_{w_{\mathrm{a} 0}} \bar{D}^{(1)}\left(\mathbf{y}_{\mathrm{r}}, \hat{\mathbf{y}}_{\mathrm{r}}\left(w_{\mathrm{a} 0}\right)\right)\right]+P\left[\cup_{w_{\mathrm{b} 0}} \bar{D}^{(2)}\left(\mathbf{y}_{\mathrm{r}}, \hat{\mathbf{y}}_{\mathrm{r}}\left(w_{\mathrm{b} 0}\right)\right)\right]+P\left[\cup_{w_{\mathrm{a} 2}, w_{\mathrm{b} 2}} \bar{D}^{(3)}\left(\mathbf{u}_{\mathrm{a}}\left(w_{\mathrm{a} 2}\right), \mathbf{u}_{\mathrm{r}}\left(w_{\mathrm{b} 2}\right)\right)\right]+ \\
& P\left[\bar{D}^{(3)}\left(\mathbf{u}_{\mathrm{b}}\left(w_{\mathrm{b} 2}\right), \mathbf{y}_{\mathrm{a}}\right)\right]+P\left[\cup_{\tilde{w}_{\mathrm{b} 2} \neq w_{\mathrm{b} 2}} D^{(3)}\left(\mathbf{u}_{\mathrm{b}}\left(\tilde{w}_{\mathrm{b} 2}\right), \mathbf{y}_{\mathrm{a}}\right)\right] \\
\leq & 4 \epsilon+2^{n\left(R_{\mathrm{b} 2}-\Delta_{3, n} I\left(U_{\mathrm{b}}^{(3)} ; Y_{\mathrm{a}}^{(3)}\right)+3 \epsilon\right)},
\end{aligned}
$$

and

$$
\begin{aligned}
P\left[E_{\mathrm{r}, \mathrm{a}}^{(4)} \mid \bar{E}_{\mathrm{r}, \mathrm{a}}^{(3)}\right] \leq & P\left[\bar{D}^{(4)}\left(\mathbf{x}_{\mathrm{r}}\left(w_{\mathrm{a} 0}, w_{\mathrm{b} 0}\right), \mathbf{y}_{\mathrm{a}}\right)\right]+ \\
& P\left[\bigcup_{\substack{\tilde{w}_{\mathrm{a} 0} \neq w_{\mathrm{b} 0} 0 \\
\text { a }}} D^{(4)}\left(\mathbf{x}_{\mathrm{r}}\left(\tilde{w}_{\mathrm{a} 0}, \tilde{w}_{\mathrm{b} 0}\right), \mathbf{y}_{\mathrm{a}}\right), D^{(1)}\left(\mathbf{x}_{\mathrm{a}}\left(w_{\mathrm{a}}\right), \hat{\mathbf{y}}_{\mathrm{r}}\left(\tilde{w}_{\mathrm{a} 0}\right)\right),\right. \\
& \left.D^{(2)}\left(\hat{\mathbf{y}}_{\mathrm{r}}\left(\tilde{w}_{\mathrm{b} 0}\right), \mathbf{y}_{\mathrm{a}}\right), s_{\mathrm{b} 0}\left(\tilde{w}_{\mathrm{b} 0}\right)=w_{\mathrm{b} 1}\right]+ \\
& P\left[\cup_{\tilde{w}_{\mathrm{b} 0} \neq w_{\mathrm{b} 0}} D^{(4)}\left(\mathbf{x}_{\mathrm{r}}\left(w_{\mathrm{a} 0}, \tilde{w}_{\mathrm{b} 0}\right), \mathbf{y}_{\mathrm{a}}\right), D^{(2)}\left(\hat{\mathbf{y}}_{\mathrm{r}}\left(\tilde{w}_{\mathrm{b} 0}\right), \mathbf{y}_{\mathrm{a}}\right), s_{\mathrm{b} 0}\left(\tilde{w}_{\mathrm{b} 0}\right)=w_{\mathrm{b} 1}\right] \\
\leq & \epsilon+2^{n\left(R_{\mathrm{a} 0}+R_{\mathrm{b} 0}-\Delta_{4, n} I\left(X_{\mathrm{r}}^{(4)} ; Y_{\mathrm{a}}^{(4)}\right)-\Delta_{1, n} I\left(\hat{Y}_{\mathrm{r}}^{(1)} ; X_{\mathrm{a}}^{(1)}\right)-\Delta_{2, n} I\left(\hat{Y}_{\mathrm{r}}^{(2)} ; Y_{\mathrm{a}}^{(2)}\right)-\alpha_{\mathrm{b}} R_{\mathrm{b} 0}+\epsilon^{\prime \prime}\right)}+ \\
& 2^{n\left(R_{\mathrm{b} 0}-\Delta_{4, n} I\left(X_{\mathrm{r}}^{(4)} ; Y_{\mathrm{a}}^{(4)}\right)-\Delta_{2, n} I\left(\hat{Y}_{\mathrm{r}}^{(2)} ; Y_{\mathrm{r}}^{(2)}\right)-\alpha_{\mathrm{b}} R_{\mathrm{b} 0}+\epsilon^{\prime \prime \prime}\right)} .
\end{aligned}
$$


In (84), the bound for $R_{\mathrm{b} 0}$ in the second term is implied by that in the third term since $R_{\mathrm{a} 0}-$ $\Delta_{1, n} I\left(\hat{Y}_{\mathrm{r}}^{(1)} ; X_{\mathrm{a}}^{(1)}\right)=\Delta_{1, n} I\left(Y_{\mathrm{r}}^{(1)} ; \hat{Y}_{\mathrm{r}}^{(1)} \mid X_{\mathrm{a}}^{(1)}\right)+\epsilon \geq 0$. Thus,

$$
\begin{aligned}
P\left[E_{\mathrm{b}, \mathrm{a}}^{(4)} \mid \bar{E}_{\mathrm{r}, \mathrm{a}}^{(3)} \cap \bar{E}_{\mathrm{r}, \mathrm{a}}^{(4)}\right] & \leq P\left[\bar{D}^{(2)}\left(\mathbf{x}_{\mathrm{b}}\left(w_{\mathrm{b}}\right), \mathbf{y}_{\mathrm{b}}, \hat{\mathbf{y}}_{\mathrm{r}}\left(w_{\mathrm{b} 0}\right)\right)\right]+P\left[\cup_{\tilde{w}_{\mathrm{b}} \neq w_{\mathrm{b}}} D^{(2)}\left(\mathbf{x}_{\mathrm{b}}\left(\tilde{w}_{\mathrm{b}}\right), \mathbf{y}_{\mathrm{b}}, \hat{\mathbf{y}}_{\mathrm{r}}\left(w_{\mathrm{b} 0}\right)\right)\right] \\
& \leq \epsilon+2^{n\left(R_{\mathrm{b}}-\Delta_{2, n} I\left(X_{\mathrm{b}}^{(2)} ; \hat{Y}_{\mathrm{r}}^{(2)}, Y_{\mathrm{a}}^{(2)}\right)+3 \epsilon\right)}
\end{aligned}
$$

Since $\epsilon>0$ is arbitrary, a proper choice of $\alpha_{\mathrm{b}}$, the conditions of Theorem 1, (77), and the AEP property guarantee that the right hand sides of (82), (84) and (86) corresponding to (77), (7) and (2) vanish as $n \rightarrow \infty$. Similarly, $P\left[E_{\mathrm{a}, \mathrm{b}}\right] \rightarrow 0$ as $n \rightarrow \infty$. By the Carathéodory theorem in [11], it is sufficient to restrict $|\mathcal{Q}| \leq 13$ since the number of corresponding mutual information terms in Theorem 1 is thirteen. Similarly, $\left|\hat{\mathcal{Y}}_{\mathrm{r}}\right| \leq|\mathcal{Q}|\left|\mathcal{Y}_{\mathrm{r}}\right|+3$. A more detailed argument of the cardinality bounds may be found in Appendix $\mathrm{C}$ in [6]. To apply a coded time sharing random variable $Q$, generate random sequences q of length $n$ i.i.d. according to $p(q)$. Then define $\mathbf{q}^{(\ell)}$ as the length $n \cdot \Delta_{\ell, n}$ sequence $\left(q^{n \cdot \sum_{i=1}^{\ell-1} \Delta_{i, n}+1}, \cdots, q^{n \cdot \sum_{i=1}^{\ell} \Delta_{i, n}}\right)$, such that $\mathbf{q}=\left(\mathbf{q}^{(1)}, \cdots, \mathbf{q}^{(4)}\right)$. We then employ coded time sharing with $\mathbf{q}^{(\ell)}$ for phase $\ell$ in the manner of [10].

\section{REFERENCES}

[1] R. Ahlswede, N. Cai, S.-Y. R. Li, and R. W. Yeung, "Network information flow," IEEE Trans. Inform. Theory, vol. 46, pp. 1204-1216, 2000.

[2] T. Berger, Multiterminal source coding. New York: Springer Verlag, 1977.

[3] M. Costa, "Writing on dirty paper," IEEE Trans. Inform. Theory, vol. IT-29, pp. 439-441, May 1983.

[4] T. Cover and J. Thomas, Elements of Information Theory, 2nd ed. New York:Wiley, 2006.

[5] T. Cover and A. El Gamal, "Capacity theorems for the relay channel," IEEE Trans. Inform. Theory, vol. IT-25, pp. 572-584, Sep. 1979.

[6] A. El Gamal and Y. Kim, "Lecture notes on network information theory," Jan. 2010, http://arxiv.org/PS_cache/arxiv/pdf/1001/1001.3404v4.pdf.

[7] A. El Gamal, M. Mohseni, and S. Zahedi, "Bounds on capacity and minimum energy-per-bit for awgn relay channels," IEEE Trans. Inform. Theory, vol. 52, no. 4, pp. 1545-1561, Apr. 2006.

[8] A. El Gamal and E. van der Meulen, "A proof of marton's coding theorem for the discrete memoryless broadcast channel," IEEE Trans. Inform. Theory, vol. IT-27, pp. 120-122, Jan. 1981.

[9] D. Gunduz, E. Tuncel, and J. Nayak, "Rate regions for the seperated two-way relay channel," in 46th Annual Allerton Conference on Communication, Control, and Computing, Monticello, IL, Sep. 2008.

[10] T. S. Han and K. Kobayashi, "A new achievable rate region for the interference channel," IEEE Trans. Inform. Theory, vol. 27, no. 1, pp. 49-60, 1981.

[11] J. B. Hiriart-Urruty and C. Lemaréchal, Fundamentals of Convex Analysis. New York; Springer, 2001.

[12] W. Kang and G. Kramer, "Broadcast channel with degraded source random variables and receiver side information," in Information Theory, 2008. ISIT 2008. IEEE International Symposium on. IEEE, 2008, pp. 1711-1715. 
[13] S. J. Kim, P. Mitran, and V. Tarokh, "Performance bounds for bi-directional coded cooperation protocols," IEEE Trans. Inform. Theory, vol. 54, no. 11, pp. 5235-5241, Nov. 2008.

[14] G. Kramer, M. Gastpar, and P. Gupta, "Cooperative strategies and capacity theorems for relay networks," IEEE Trans. Inform. Theory, vol. 51, no. 9, pp. 3037-3063, Sep. 2005.

[15] P. Larsson, N. Johansson, and K.-E. Sunell, "Coded bi-directional relaying," in Proc. IEEE Veh. Technol. Conf. - Spring, 2006, pp. 851-855.

[16] K. Marton, "A coding theorem for the discrete memoryless broadcast channel," IEEE Trans. Inform. Theory, vol. 25, pp. 306-311, May 1979.

[17] W. Nam, S.-Y. Chung, and Y. Lee, "Capacity of the Gaussian two-way relay channel to within 1/2 bit," 2009. [Online]. Available: http://arxiv.org/abs/0902.2438

[18] K. Narayanan, M. P. Wilson, and A. Sprintson, "Joint physical layer coding and network coding for bi-directional relaying," in 45th Annual Allerton Conference, Monticello, IL, Sep. 2007.

[19] J. Nayak, E. Tuncel, and D. Gunduz, "Wyner-ziv coding over broadcast channels," in IEEE Inform. Theory Workshop, Porto, Portugal, Jun. 2008.

[20] B. Nazer and M. Gastpar, "Compute-and-forward: Harnessing interference through structured codes," Arxiv preprint arXiv:0908.2119, 2009.

[21] T. J. Oechtering, C. Schnurr, I. Bjelakovic, and H. Boche, "Achievable rate region of a two phase bidirectional relay channel," in Proc. Conf. on Inf. Sci. and Sys., Baltimore, MD, Mar. 2007.

[22] T. Oechtering, I. Bjelakovic, C. Schnurr, and H. Boche, "Broadcast capacity region of two-phase bidirectional relaying," Arxiv preprint cs/0703078, 2007.

[23] Y. Oohama, "Gaussian multiterminal source coding," IEEE Trans. Inform. Theory, vol. 43, no. 6, pp. 1912-1923, Nov. 1997.

[24] P. Popovski and H. Yomo, "The anti-packets can increase the achievable throughput of a wireless multi-hop network," in Proc. IEEE Int. Conf. Commun., 2006, pp. 3885-3890.

[25] — - "Bi-directional amplification of throughput in a wireless multi-hop network," in Proc. IEEE Veh. Technol. Conf. Spring, 2006, pp. 588-593.

[26] B. Rankov and A. Wittneben, "Spectral efficient signaling for half-duplex relay channels," in 39th Asilomar Conference on Signals, Systems, and Computers, Pacific Grove, CA, Nov. 2005.

[27] C. Schnurr, T. J. Oechtering, and S. Stanczak, "Achievable rates for the restricted half-duplex two-way relay channel," in 41st Asilomar Conference on Signals, Systems and Computers, Monterey, CA, Nov. 2007, pp. 1468-1472.

[28] C. Schnurr, S. Stanczak, and T. J. Oechtering, "Achievable rates for the restricted half-duplex two-way relay channel under a partial-decode-and forword protocol," in Proc. IEEE Inf. Theory Workshop, Porto, Portugal, May 2008, pp. 134-138.

[29] — - "Coding theorems for for the restricted half-duplex two-way relay channel with joint decoding," in Proc. IEEE Int. Symp. Inform. Theory, Toronto, Canata, Jul. 2008, pp. 2688-2692.

[30] Y. Song and N. Devroye, "Lattice list decoding and applications to relay channels," in 48th Annual Allerton Conference, Monticello, IL, Sep. 2010.

[31] E. Tuncel, "Slepian-wolf coding over broadcast channels," IEEE Trans. Inform. Theory, vol. 52, no. 4, pp. 1469-1482, Apr. 2006.

[32] L. L. Xie, "Network coding and random binning for multi-user channels," in the 10th Canadian Workshop on Information Theory, Edmonton, Alberata, Canada, Jun. 2007, pp. 85 - 88. 
[33] R. Zamir, "Lattices are everywhere," in 4th Annual Workshop on Information Theory and its Applications, UCSD, 2009.

\begin{tabular}{|l|}
\hline \\
PLACE \\
PHOTO \\
HERE
\end{tabular}

perspective.
Sang Joon Kim received the Bachelor's degree in electrical engineering from Seoul National University, Seoul, Korea(ROK), in 2001 and the Master's degree in Applied Mathematics and the Ph.D. in Engineering Science from School of Engineering and Applied Sciences, Harverd University, Cambridge, MA, in 2007 and 2009, respectively. In 2001-2005 he worked at Xeline, Co., Ltd. as a senior researcher and since 2009, he has been with Samsung Advanced Institute of Technologies as a Research staff member. His current research interests include multi-user wireless systems from information theory and coding theory

Natasha Devroye has been an Assistant Professor in the Department of Electrical and Computer Engineering at the University of Illinois at Chicago since January 2009. From July 2007 until July 2008 she was a Lecturer at Harvard University. Dr. Devroye obtained her Ph.D in Engineering Sciences from the School of Engineering and Applied Sciences at Harvard University in 2007, an M.Sc from Harvard University in 2003 and a Honors B. Eng in Electrical Engineering from McGill University in 2001. Dr. Devroye was a recipient of an NSF CAREER award in 2011. Her research focuses on multi-user information theory and applications to cognitive and software-defined radio, radar, two-way and wireless communications in general.

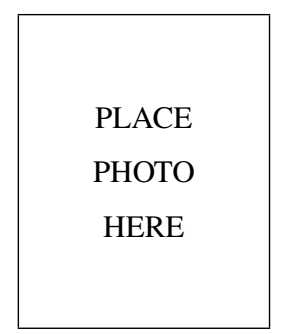

Patrick Mitran (S'01, M'07) received the Bachelor's and Master's degrees in electrical engineering, in 2001 and 2002, respectively, from McGill University, Montreal, PQ, Canada, and the Ph.D. degree from the Division of Engineering and Applied Sciences, Harvard University, Cambridge, MA in 2006. In 2005, he interned as a research scientist for Intel Corporation in the Radio Communications Lab. In 2006-07 he was an applied mathematics lecturer in the School of Engineering and Applied Sciences, Harvard University. Since fall 2007, he is with the Department of Electrical and Computer Engineering at the University of Waterloo at the rank of Assistant Professor.

Currently he is interested in the study of cooperation and cognition in wireless networks from signal processing, coding theory an information theory perspectives.

June 14, 2011 


\begin{tabular}{|c|}
\hline \\
PLACE \\
PHOTO \\
HERE \\
\hline
\end{tabular}

Vahid Tarokh received the Ph.D. degree in Electrical Engineering in 1995. He worked at AT\&T LabsResearch and AT\&T Wireless Services until 2000 where he was (inchronological order) Senior Member of Technical Staff, Principal Member of Technical Staff and Head of Department of Wireless Communications and Signal Processing. In 2000, he joined the Electrical Engineering Department at MIT as an Associate Professor. He then joined Harvard faculty and was appointed a Gordon MacKay Professor of Electrical Engineering in 2002. He was named Perkins Professor and Vinton Hayes Senior Research Fellow of Electrical Engineering in 2005. Dr. Tarokh has received a number of awards and holds 2 honorary degrees. 